\title{
INFRARED AND RADIO OBSERVATIONS OF A SMALL GROUP OF PROTOSTELLAR OBJECTS IN THE MOLECULAR CORE, L1251-C
}

\author{
Jungha Kim ${ }^{1}$, Jeong-Eun Lee ${ }^{1,4}$, Minho Chol $^{2}$, Tyler L. Bourke ${ }^{3}$, Neal J. Evans $\mathrm{II}^{4}$, \\ James Di Francesco $^{5}$, Lucas A. Cieza ${ }^{6}$, Michael M. Dunham ${ }^{7}$, and Miju Kang ${ }^{2}$ \\ ${ }^{1}$ School of Space Research, Kyung Hee University, Yongin-Si, Gyeonggi-Do 446-701, Korea \\ ${ }^{2}$ Korea Astronomy and Space Science Institute, 776 Daedeokdaero, Yuseong, Daejeon 305-348, Korea \\ ${ }^{3}$ Square Kilometre Array Organisation, Jodrell Bank Observatory, Lower Withington, Cheshire SK11 9DL, UK \\ ${ }^{4}$ Department of Astronomy, University of Texas at Austin, 2515 Speedway, Stop C1400, Austin, TX 78712-1205, USA \\ National Research Council Canada, Herzberg Institute of Astrophysics, Victoria, BC, Canada \\ ${ }^{6}$ Universidad Diego Portales, Facultad de Ingeniera, Av. Ejército 441, Santiago, Chile \\ ${ }^{7}$ Harvard-Smithsonian Center for Astrophysics, 60 Garden Street, Cambridge, MA 02138, USA \\ Received 2014 November 23; accepted 2015 March 10; published 2015 May 1
}

\begin{abstract}
We present a multi-wavelength observational study of a low-mass star-forming region, L1251-C, with observational results at wavelengths from the near-infrared to the millimeter. Spitzer Space Telescope observations confirmed that IRAS $22343+7501$ is a small group of protostellar objects. The extended emission in the east-west direction with its intensity peak at the center of L1251A has been detected at 350 and $850 \mu \mathrm{m}$ with the Caltech Submillimeter Observatory and James Clerk Maxwell telescopes, tracing dense envelope material around L1251A. The single-dish data from the Korean VLBI Network and TRAO telescopes show inconsistencies between the intensity peaks of several molecular emission lines and that of the continuum emission, suggesting complex distributions of molecular abundances around L1251A. The Submillimeter Array interferometer data, however, show intensity peaks of CO 2-1 and ${ }^{13} \mathrm{CO} 2-1$ located at the position of IRS 1, which is both the brightest source in the Infrared Array Camera image and the weakest source in the $1.3 \mathrm{~mm}$ dust-continuum map. IRS 1 is the strongest candidate for the driving source of the newly detected compact CO 2-1 outflow. Over the entire region $\left(14^{\prime} \times 14^{\prime}\right)$ of L125l-C, 3 Class I and 16 Class II sources have been detected, including three young stellar objects (YSOs) in L1251A. A comparison between the average projected distance among the 19 YSOs in L1251-C and that among the 3 YSOs in L1251A suggests that L1251-C is an example of low-mass cluster formation where protostellar objects form in a small group.
\end{abstract}

Key words: ISM: individual objects (L1251-C) - ISM: jets and outflows - stars: formation - stars: protostars

\section{INTRODUCTION}

Many studies of low-mass star formation have been conducted in detail. Star formation mostly occurs in groups and clusters where the number density of young stellar objects (YSOs) is at least $\sim 3 \mathrm{pc}^{-2}$ (Lada \& Lada 2003). The Spitzer legacy project "From Molecular Cores to Planets Forming Disks" (c2d; Evans et al. 2003, 2009) found that most sites of low-mass star formation within $400 \mathrm{pc}$ have significant populations of protostars forming in small groups of multiple objects (e.g., <10 members) located in close proximity.

L1251 is a star-forming region located in Cepheus at a distance of $300 \pm 50 \mathrm{pc}$ (Kun \& Prusti 1993) from the Sun. There are five dense $\mathrm{C}^{18} \mathrm{O}$ cores A-E $\left(\mathrm{N}\left(\mathrm{C}^{18} \mathrm{O}\right) \sim 10^{15} \mathrm{~cm}^{-2}\right)$ in L1251 which have been assigned A-E with R.A. (Sato et al. 1994). Two outflows, an extended CO 1-0 outflow and a well-collimated compact CO 1-0 outflow were previously known in cores C and E, respectively (Sato \& Fukui 1989). These two outflows have been named L1251A and L1251B by Hodapp (1994). Core $\mathrm{C}$ is the second densest $\mathrm{C}^{18} \mathrm{O}$ core among the five cores in L1251 (Sato et al. 1994) where the strongest extended CO 1-0 outflow (L1251A) is located (Sato \& Fukui 1989). The dense region around IRAS 22376+7455, which is the driving source of the well-collimated compact outflow (L1251B) in core E, has been studied in detail (Lee et al. 2006, 2007). Recently, another well-collimated outflow in core A was revealed (Lee et al. 2010). In this paper, we use "L1251-C" to present the extended region observed in $\mathrm{C}^{18} \mathrm{O}$
$1-0$ which is the same region as "L1251C" in the Spitzer data archive. On the other hand, "L1251A" is used to refer to the densest region around IRAS $22343+7501$, which is believed to be the driving source of the extended CO 2-1 outflow. Our Spitzer observations revealed that L1251A is a multiprotostellar system consisting of three sources.

Earlier studies have already shown that IRAS $22343+7501$ is composed of multiple protostellar objects. Rosvick \& Davidge (1995) found that there are five near-infrared (NIR) sources, labeled A-E, around L1251A. Meehan et al. (1998), Beltrán et al. (2001), and Reipurth et al. (2004) also presented multiple radio continuum sources detected with the Very Long Array (VLA) in L1251A. Two VLA radio sources, VLA 6 and VLA 7 (Beltrán et al. 2001; Reipurth et al. 2004), are associated with the NIR sources D and A of Rosvick \& Davidge (1995), respectively. Kun et al. (2009) presented optical spectra and photometry for sources A and B of Rosvick \& Davidge (1995) and classified both sources as classical T Tauri stars.

$\mathrm{NH}_{3}$ cores were also detected around L1251A (Anglada et al. 1997; Toth \& Walmsley 1996). Nikolić et al. (2003) presented the distributions of several molecular transitions compared with the $\mathrm{NH}_{3}$ distribution of Toth \& Walmsley (1996) and showed outflow-like structures which may be driven by VLA 6 and VLA 7 in $\mathrm{HCO}^{+} 1-0 . \mathrm{H}_{2} \mathrm{O}$ masers were also detected (Toth \& Walmsley 1994; Wilking et al. 1994; Xiang \& Turner 1995; Claussen et al. 1996) in this region.

Schwartz et al. (1988) discovered a bipolar CO 1-0 outflow in L1251A. This CO 1-0 bipolar outflow shows asymmetric 
Table 1

Summary of Radio Single-dish Observations

\begin{tabular}{|c|c|c|c|c|c|c|}
\hline Molecular Line & $\begin{array}{c}\nu \\
(\mathrm{GHz})\end{array}$ & $\begin{array}{c}\Delta V \\
\left(\mathrm{~km} \mathrm{~s}^{-1}\right)\end{array}$ & $\begin{array}{l}\text { Beam FWHM } \\
(\operatorname{arcsec})\end{array}$ & $\eta_{\mathrm{mb}}$ & $\begin{array}{c}1 \sigma \mathrm{rms} \\
(\mathrm{K})\end{array}$ & Telescope \\
\hline \multirow[t]{2}{*}{$\mathrm{H}_{2} \mathrm{O} 6_{16}-5_{23}$} & 22.235080 & 0.21 & 120 & 0.38 & 0.08 & KVN (YS) \\
\hline & $\ldots$ & $\ldots$ & $\ldots$ & 0.42 & 0.09 & KVN (US) \\
\hline $\mathrm{H}^{13} \mathrm{CO}^{+} 1-0$ & 86.754288 & 0.22 & 31.5 & 0.42 & 0.08 & KVN \\
\hline $\mathrm{HCN} 1-0$ & 88.631847 & 0.22 & 31.5 & 0.41 & 0.09 & KVN \\
\hline $\mathrm{HCO}^{+} 1-0$ & 89.188526 & 0.22 & 31.5 & 0.40 & 0.09 & KVN \\
\hline $\mathrm{N}_{2} \mathrm{H}^{+} 1-0$ & 93.176265 & 0.22 & 31.5 & 0.37 & 0.09 & KVN \\
\hline $\mathrm{H}_{2} \mathrm{CO} 2_{12}-1_{11}$ & 140.839520 & 0.27 & 23.4 & 0.30 & 0.11 & $\mathrm{KVN}$ \\
\hline CO 1-0 & 115.271202 & 0.21 & 50 & 0.48 & 0.37 & TRAO \\
\hline
\end{tabular}

blue and red lobes in morphology and intensity. Sato \& Fukui (1989) also detected an extended CO 1-0 outflow in L1251A with a symmetric structure. Interestingly, the CO 1-0 outflow from Sato \& Fukui (1989) has a northeast-southwest (NESW) axis, which is perpendicular to the northwest-southeast (NW-SE) axis found by Schwartz et al. (1988). An optical jet was found by Balazs et al. (1992) near L1251A, supporting L1251A as the origin of the extended CO 1-0 outflow.

In this paper, we analyze the properties of YSOs based on the $\mathrm{c} 2 \mathrm{~d}$ survey data and newly obtained molecular line data to study the environment of star formation in L1251-C, especially around L1251A. In this study, we use the $(\alpha, \delta)$ coordinates of IRAS $22343+7501$ as $\left(22^{\mathrm{h}} 35^{\mathrm{m}} 23^{\mathrm{s}} \cdot 4,+75^{\circ} 17^{\prime} 07^{\prime \prime}\right)$ in the J2000.0 epoch.

Our observations of L1251-C are summarized in Section 2. Our results and analysis of the data are described in Sections 3 and 4, respectively. In Section 5, we discuss star formation in small groups and outflows in L1251A. Finally, a summary of this paper is provided in Section 6.

\section{OBSERVATIONS AND DATA}

\subsection{Spitzer Space Telescope Data}

In the Spitzer legacy program c2d, L1251-C was observed at 3.6, 4.5, 5.8, and $8.0 \mu \mathrm{m}$ with the Infrared Array Camera (IRAC; Fazio et al. 2004) on 2004 October 18 and was also observed at 24 and $70 \mu \mathrm{m}$ with the Multi-band Imaging Photometer for Spitzer (MIPS; Rieke et al. 2004) on 2004 September 24. The High-dynamic Range (HDR) mode observations were obtained to provide more accurate intensity levels of saturated sources. In the HDR mode, a longintegration frame of $10.4 \mathrm{~s}$ and a short-integration frame of $0.4 \mathrm{~s}$ were taken at each position. The description of the data processing details can be found in the c2d final delivery documentation (see Evans et al. 2007). ${ }^{8}$ In this paper, we use the c2d photometric data catalog of YSOs including 2MASS photometric data. For the saturated sources, however, we use the photometry obtained with the HDR mode data.

\subsection{Radio Single-dish Observations and Data}

\subsubsection{Korean VLBI Network (KVN) Observations}

Molecular line observations of L1251-C were carried out with the KVN $21 \mathrm{~m}$ radio telescope at the Yonsei site, Seoul from 2012 October to 2013 March and at the Ulsan site, Ulsan in 2013 April. The central position of mapping observations

\footnotetext{
8 See http://irsa.ipaccaltech.edu/data/SPITZER/docs/spitzermission/observingprograms/ legacy/
}

was the position of IRAS $22343+7501$. We observed the $\mathrm{HCO}^{+} 1-0, \mathrm{H}^{13} \mathrm{CO}^{+} 1-0, \mathrm{~N}_{2} \mathrm{H}^{+} 1-0$, and $\mathrm{HCN} 1-0$ lines to map the cloud structure. We also simultaneously observed the $\mathrm{H}_{2} \mathrm{O} 6_{16}-5_{23}$ maser and the $\mathrm{H}_{2} \mathrm{CO} 2_{12}-1_{11}$ transition. The KVN radio telescopes were designed for simultaneous observations in the 22, 43, 86, and $129 \mathrm{GHz}$ bands (Han et al. 2008). The pointing was checked every $2 \mathrm{hr}$ using $\mathrm{R}$ Cas, a strong $\mathrm{SiO}$ maser source near the target source.

The system temperatures during the observations ranged from 60 to $250 \mathrm{~K}$ (at $22 \mathrm{GHz}$ ), from 100 to $250 \mathrm{~K}$ (at $86 \mathrm{GHz}$ ), and from 150 to $400 \mathrm{~K}$ (at $129 \mathrm{GHz}$ ), depending on weather conditions and elevations. We used the digital spectrometer in its $32 \mathrm{MHz}$ mode, providing velocity resolutions of $0.21 \mathrm{~km} \mathrm{~s}^{-1}$ (4096 channels at $22 \mathrm{GHz}$ ), $0.21 \mathrm{~km} \mathrm{~s}^{-1}$ (4096 channels at $86 \mathrm{GHz}$ ), and $0.27 \mathrm{~km} \mathrm{~s}^{-1}$ (4096 channels at $\left.129 \mathrm{GHz}\right)$. The $1 \sigma$ rms noise levels are presented in Table 1. The data were calibrated using the chopper wheel method to obtain the antenna temperature $T_{A}^{*}$.

The beam FWHM is $\sim 30^{\prime \prime}$ at $86 \mathrm{GHz}$. The maps were sampled at every half of the beam FWHM $\left(15^{\prime \prime}\right)$. We used the $\mathrm{CLASS}^{9}$ reduction package from the Institue de Radioastronomi Millimétrique (IRAM) for data reduction. A summary of observations is presented in Table 1 .

\subsubsection{Taeduk Radio Astronomy Observatory (TRAO) Observations}

Observations of CO 1-0 were carried out in 2013 February with the $14 \mathrm{~m}$ radio telescope of TRAO, equipped with nine receivers arranged in a $3 \times 3$ pattern with $50^{\prime \prime}$ spacing. The central position of the target was the same as in the KVN observations. The system temperatures during observations ranged from 950 to $1100 \mathrm{~K}$ depending on weather conditions and source elevations. The velocity resolution is $0.327 \mathrm{~km} \mathrm{~s}^{-1}$ and the averaged $1 \sigma \mathrm{rms}$ noise level is about $0.37 \mathrm{~K}$.

The beam FWHM and beam efficiency are $50^{\prime \prime}$ and 0.48 at $115 \mathrm{GHz}$, respectively. We mapped a $\sim 275^{\prime \prime} \times 275^{\prime \prime}$ region around L1251A, sampling at every half of the beam FWHM $\left(25^{\prime \prime}\right)$. We also used the CLASS reduction package from IRAM for data reduction. A summary of observations is also presented in Table 1.

\subsubsection{Data from Other Observations}

The $350 \mu$ m continuum emission map (Wu et al. 2007) of L1251-C was obtained using the SHARC-II camera on the Caltech Submillimeter Observatory (CSO).

\footnotetext{
9 See http://www.iram.fr/IRAMFR/GILDAS/doc/html/class-html/class.html
} 
Table 2

Summary of SMA Observations

\begin{tabular}{lccccccc}
\hline \hline Molecular Line & $\begin{array}{c}\nu \\
(\mathrm{GHz})\end{array}$ & Side Band & $\begin{array}{c}\text { Number of } \\
\text { Channels }\end{array}$ & $\begin{array}{c}\Delta V \\
\left(\mathrm{~km} \mathrm{~s}^{-1}\right)\end{array}$ & $\begin{array}{c}\text { Beam FWHM } \\
(\operatorname{arcsec} \times \operatorname{arcsec})\end{array}$ & $\begin{array}{c}\text { P.A. } \\
(\mathrm{deg})\end{array}$ & $\begin{array}{c}1 \sigma \text { rms } \\
\left(\mathrm{Jy} \mathrm{beam}{ }^{-1}\right)\end{array}$ \\
\hline CO 2-1 & 230.5379700 & USB & 128 & 1.06 & $3.6 \times 3.1$ & -1.3 & 0.20 \\
${ }^{13}$ CO 2-1 & 220.3986765 & LSB & 512 & 0.28 & $3.7 \times 2.3$ & -5.8 & 0.37 \\
\hline
\end{tabular}

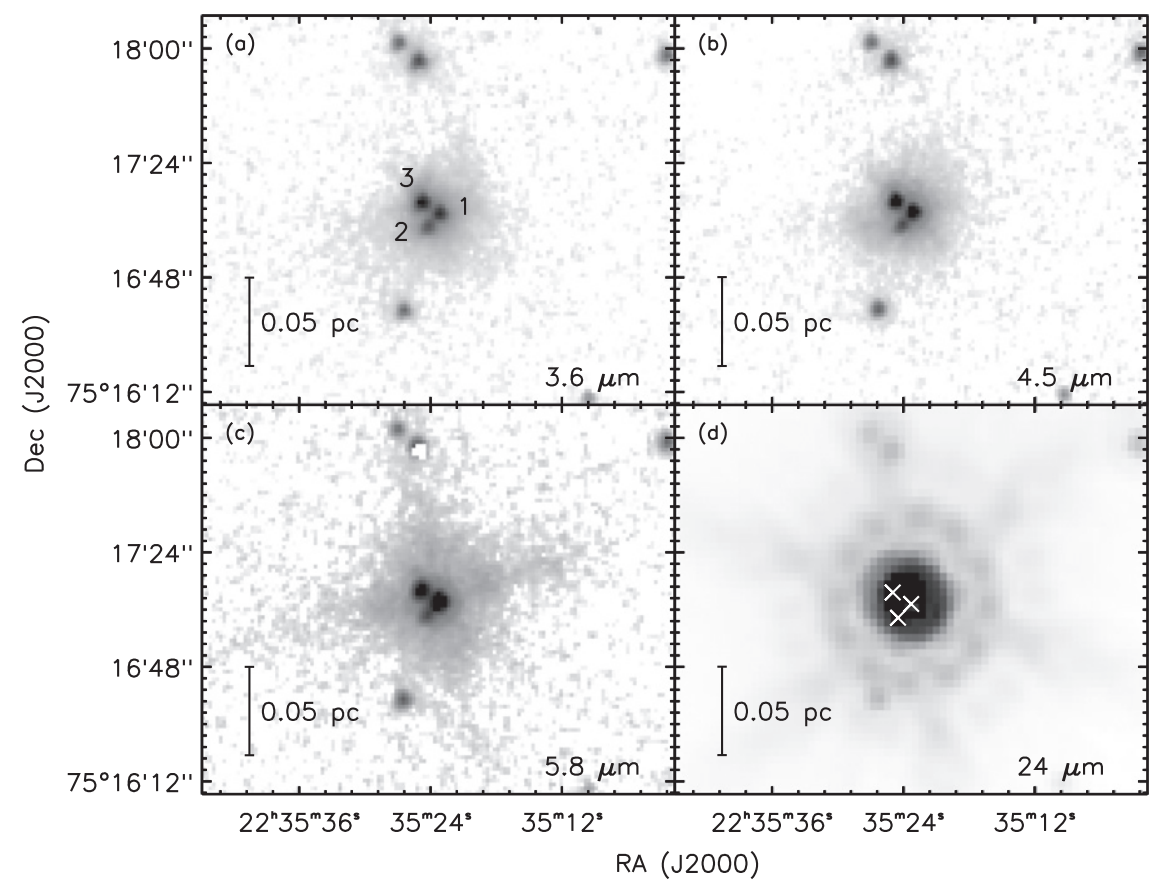

Figure 1. Spitzer images of L1251A in the log scale at (a) IRAC $3.6 \mu \mathrm{m}$, (b) IRAC $4.5 \mu \mathrm{m}$, (c) IRAC $5.8 \mu \mathrm{m}$, and (d) MIPS $24 \mu \mathrm{m}$. In Figure 1(d), the Xs indicate the positions of the IR sources in L1251A.

The $850 \mu$ m continuum emission map was reprocessed by Di Francesco et al. (2008) using the data obtained from the James Clerk Maxwell Telescope (JCMT) Submillimeter Common User Bolometer Array (SCUBA). The data used in this work are from the public archive maintained by the Canadian Astronomical Data Centre ${ }^{10}$.

\subsection{Radio Interferometer Observations}

\subsubsection{Submillimeter Array Observations}

The Submillimeter Array (SMA; Ho et al. 2004) observations of L1251A were carried out on 2007 October 17 in the compact configuration with six antennas $\left(\theta_{\mathrm{FWHM}} \sim 3^{\prime \prime}\right)$. The quasars $\mathrm{J} 2005+778$ and $\mathrm{J} 0102+584$ were observed for gain calibration. Titan was observed for flux calibration and the quasar 3C 454.3 was observed for pass-band calibration. Natural weighting was used without any tapering to produce the data cubes. The $230 \mathrm{GHz}$ receiver was tuned to observe $\mathrm{CO}$ $2-1$ and ${ }^{13} \mathrm{CO} 2-1$ in separate correlator windows of $2 \mathrm{GHz}$ bandwidths. The channel widths for $\mathrm{CO} 2-1$ and ${ }^{13} \mathrm{CO} 2-1$ are 1.06 and $0.28 \mathrm{~km} \mathrm{~s}^{-1}$, respectively. The rms noise levels of $\mathrm{CO}$ $2-1$ and ${ }^{13} \mathrm{CO} 2-1$ are presented in Table 2 . The remaining chunk of 24 windows were assigned to observe the $1.3 \mathrm{~mm}$ $(225.404 \mathrm{GHz})$ continuum emission. The continuum image was processed using the appropriate primary beam correction

\footnotetext{
${ }^{10}$ The Canadian Astronomical Data Centre is operated by the Dominion Astrophysical Observatory for the National Research Council of Canada.
}

(the primary beam is $\sim 55^{\prime \prime}$ at this frequency). The $1 \sigma$ rms level of the $1.3 \mathrm{~mm}$ continuum map is $4.5 \mathrm{mJy}^{-1}$ beam $^{-1}$. The IDLbased MIR software package ${ }^{11}$ adapted for SMA was used for calibration and MIRIAD (Sault et al. 1995) was used for imaging and analysis. A summary of observations is shown in Table 2 .

\section{RESULTS}

\subsection{Infrared Images}

\subsubsection{Central Triple System: L1251A}

IRAC 3.6, 4.5, $5.8 \mu \mathrm{m}$, and MIPS $24 \mu \mathrm{m}$ images of L1251A are presented in Figure 1. Three IR sources, IRS 1, IRS 2, and IRS 3, were identified from the HDR mode observations in the IRAC band images. On the other hand, these sources are unresolved in the MIPS band image (Figure 1(d)). Multicomponents of L1251A in the NIR were detected for the first time by Rosvick \& Davidge (1995), where five NIR sources were identified and labeled as A-E near IRAS $22343+7501$. Unfortunately, the coordinates for the five sources from Rosvick \& Davidge (1995) are not sufficiently precise to associate them with the Spitzer sources. Comparisons of the relative positions show that Spitzer sources IRS 1, IRS 2, and IRS 3 correspond to source D, source 4/B/C, and source 1/A of Rosvick \& Davidge (1995), respectively. IRS 1 is the brightest

\footnotetext{
$\overline{{ }^{11} \text { See https://www.cfa.harvard.edu/ cqi/mircook.html }}$
} 


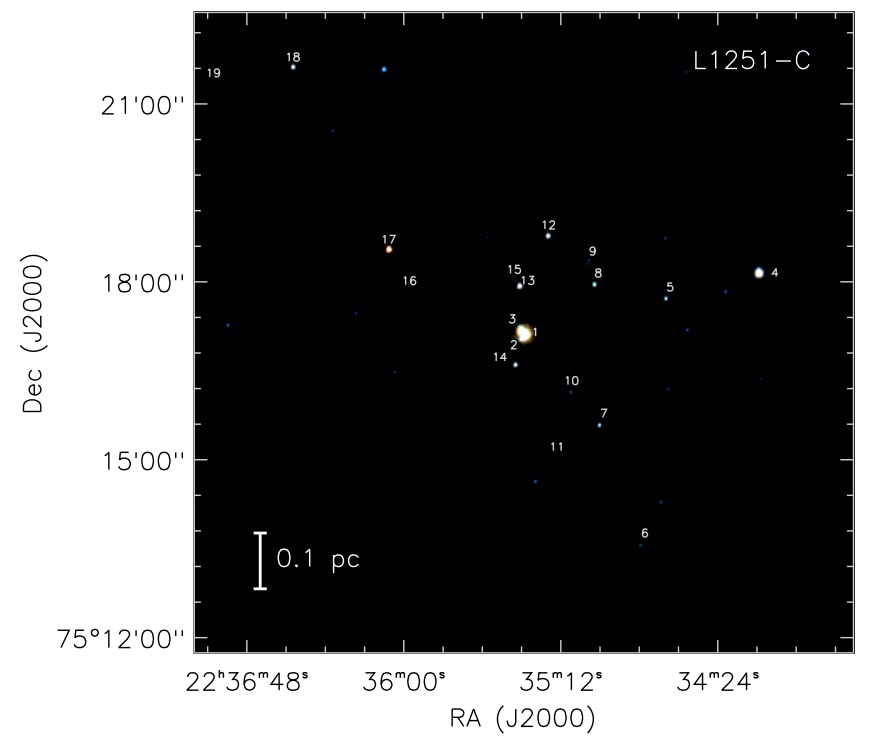

Figure 2. Spitzer three-color composite image of L1251-C. The IRAC 3.6, 4.5, and $8.0 \mu \mathrm{m}$ are presented in blue, green, and red, respectively. Numbers (4)(19) are assigned along with R.A. after the numbers (1)-(3) of three sources in L1251A, which are also assigned along with R.A.

source among three components in the IRAC band images, except in the IRAC 1 band $(3.6 \mu \mathrm{m})$, while IRS 2 is the faintest.

\subsubsection{YSO Candidates in L1251-C}

Figure 2 shows the IRAC three-color composite image of the 3.6, 4.5, and $8.0 \mu \mathrm{m}$ bands. For identification of YSO candidates, Evans et al. (2007) excluded possible extra-galactic background sources based on criteria derived using the colormagnitude diagrams of the reprocessed Spitzer Wide-area
Infrared Extragalactic Survey (Lonsdale et al. 2003) data. According to the c2d YSOc catalog, 16 YSO candidates were identified in this region. Three more sources were identified by HDR mode observations (IRS 1, IRS 2, and IRS 3). Including the three added sources, 19 YSO candidates were identified in total, and Figure 2 shows our identification numbers for the sources around L1251-C in the IRAC $4.5 \mu \mathrm{m}$ image. The labels 1,2 , and 3 represent IRS 1 , IRS 2, and IRS 3, respectively, and the labels from 4 to 19 are assigned along with R.A. coordinates and Spitzer flux densities of the YSO candidates identified in the Spitzer images are presented in Table 3.

\subsection{Continuum Images, Molecular Line Spectra, and Maps}

\subsubsection{Radio Single-dish Continuum Emission}

Figure 3 shows the continuum images observed with CSO SHARC-II at $350 \mu \mathrm{m}$ and JCMT SCUBA at $850 \mu \mathrm{m}$. Dust continuum condensations associated with L1251A have been detected in both wavelengths (Young et al. 2006; Wu et al. 2007). With effective beam FWHMs of $\sim 9^{\prime \prime}$ and $\sim 22$ !'9, respectively, the YSOs in L1251A were not resolved. The peak positions of dust-continuum emission in both wavelengths are located at the center of the three IR sources, suggesting that the dust-continuum emission traces the dense core covering L1251A with peak intensities of 5.4 and $1.02 \mathrm{Jy} \mathrm{beam}^{-1}$ at 350 and $850 \mu \mathrm{m}$, respectively. Dust continuum condensations in both wavelengths are observed to be elongated in the east-west $(\mathrm{E}-\mathrm{W})$ direction from L1251A. The $850 \mu$ m dust-continuum emission is likely optically thin and traces the dense and cold material within the molecular core. Therefore, the total (gas+dust) mass of L1251A, $M_{\text {total, was }}$

Table 3

Infrared Fluxes of Young Stellar Object Candidates from the Spitzer Data

\begin{tabular}{|c|c|c|c|c|c|c|c|c|}
\hline Source & $\begin{array}{l}\text { R.A. } \\
(\mathrm{h} \mathrm{m} \mathrm{s})\end{array}$ & $\begin{array}{l}\text { Decl. } \\
(0, \prime \prime)\end{array}$ & $\begin{array}{l}\text { IRAC } 3.6 \mu \mathrm{m} \\
(\mathrm{mJy})\end{array}$ & $\begin{array}{l}\text { IRAC } 4.5 \mu \mathrm{m} \\
(\mathrm{mJy})\end{array}$ & $\begin{array}{l}\text { IRAC } 5.8 \mu \mathrm{m} \\
(\mathrm{mJy})\end{array}$ & $\begin{array}{c}\text { IRAC } 8.0 \mu \mathrm{m} \\
(\mathrm{mJy})\end{array}$ & $\begin{array}{c}\text { MIPS } 24 \mu \mathrm{m} \\
(\mathrm{mJy})\end{array}$ & $\begin{array}{c}\text { MIPS } 70 \mu \mathrm{m} \\
(\mathrm{mJy})\end{array}$ \\
\hline 1 & $22: 35: 23.28$ & $+75: 17: 07.73$ & $293.00(13.00)$ & $748.00(13.00)$ & $1,550.00(32.00)$ & $2,510.00(39.900)$ & $\cdots$ & $\cdots$ \\
\hline 2 & $22: 35: 24.57$ & $+75: 17: 03.61$ & $183.00(3.20)$ & $114.00(3.60)$ & $111.00(3.30)$ & $128.00(1.30)$ & $\cdots$ & $\ldots$ \\
\hline 3 & $22: 35: 24.96$ & $+75: 17: 11.33$ & $532.00(22.00)$ & $610.00(11.00)$ & $707.00(14.00)$ & $890.00(19.00)$ & $\cdots$ & $\cdots$ \\
\hline 4 & $22: 34: 12.00$ & $+75: 18: 09.36$ & $183.00(58.90)$ & $114.00(14.60)$ & $111.00(34.90)$ & $128.00(31.40)$ & $1,440.00(135.00)$ & $\begin{array}{l}1,740.00 \\
(162.00)\end{array}$ \\
\hline 5 & $22: 34: 40.56$ & $+75: 17: 44.16$ & $30.20(1.51)$ & $28.40(1.38)$ & $27.70(1.36)$ & $35.60(1.40)$ & $139.00(12.90)$ & $\begin{array}{l}257.00 \\
(24.50)\end{array}$ \\
\hline 6 & $22: 34: 48.24$ & $+75: 13: 35.04$ & $8.97(0.44)$ & $7.30(0.35)$ & $6.68(0.32)$ & $9.04(0.43)$ & $17.80(1.66)$ & $50.90(5.83)$ \\
\hline 7 & $22: 35: 00.72$ & $+75: 15: 36.36$ & $22.00(1.13)$ & $23.40(1.13)$ & $25.70(1.25)$ & $32.30(1.52)$ & $102.00(9.44)$ & $\begin{array}{r}146.00 \\
(14.50)\end{array}$ \\
\hline 8 & $22: 35: 02.40$ & $+75: 17: 58.20$ & $35.60(1.83)$ & $43.10(2.13)$ & $45.60(2.23)$ & $47.60(2.30)$ & $65.30(6.06)$ & $\ldots$ \\
\hline 9 & $22: 35: 04.08$ & $+75: 18: 20.52$ & $32.40(1.64)$ & $32.00(1.54)$ & $36.00(1.74)$ & $40.80(1.95)$ & $80.30(7.44)$ & $\begin{array}{l}106.00 \\
(10.70)\end{array}$ \\
\hline 10 & $22: 35: 09.60$ & $+75: 16: 09.48$ & $7.96(0.40)$ & $8.40(0.41)$ & $6.19(0.32)$ & $5.13(0.24)$ & $12.70(1.19)$ & $\ldots$ \\
\hline 11 & $22: 35: 14.06$ & $+75: 15: 02.39$ & $1.50(0.08)$ & $1.69(0.11)$ & $2.56(0.12)$ & $20.00(1.86)$ & $146.00(14.30)$ & $\cdots$ \\
\hline 12 & $22: 35: 16.56$ & $+75: 18: 46.80$ & $75.90(4.02)$ & $74.20(3.81)$ & $73.00(3.64)$ & $85.90(4.15)$ & $107.00(9.96)$ & $58.50(6.78)$ \\
\hline 13 & $22: 35: 25.44$ & $+75: 17: 56.04$ & $102.00(5.39)$ & $108.00(5.38)$ & $121.00(6.05)$ & $123.00(5.97)$ & $75.50(7.45)$ & $\ldots$ \\
\hline 14 & $22: 35: 26.62$ & $+75: 16: 36.86$ & $34.50(1.78)$ & $53.20(2.64)$ & $58.00(2.81)$ & $54.10(2.71)$ & $67.90(8.19)$ & $\cdots$ \\
\hline 15 & $22: 35: 27.12$ & $+75: 18: 01.80$ & $37.20(1.85)$ & $34.20(1.66)$ & $33.00(1.64)$ & $3.55(1.70)$ & $52.90(5.25)$ & $\cdots$ \\
\hline 16 & $22: 35: 59.21$ & $+75: 17: 50.40$ & $0.071(0.007)$ & $0.078(0.007)$ & $0.084(0.024)$ & $0.118(0.020)$ & $7.820(1.760)$ & $\cdots$ \\
\hline 17 & $22: 36: 05.52$ & $+75: 18: 32.04$ & $78.20(4.11)$ & $102.00(5.30)$ & $145.00(7.29)$ & $246.00(12.20)$ & $534.00(45.60)$ & 377. (36.60) \\
\hline 18 & $22: 36: 35.52$ & $+75: 21: 34.92$ & $54.80(2.72)$ & $54.90(2.77)$ & $58.10(2.81)$ & $72.60(3.65)$ & $206.00(19.10)$ & $\cdots$ \\
\hline 19 & $22: 36: 59.04$ & $+75: 21: 20.52$ & $2.87(0.19)$ & $2.38(0.14)$ & $2.11(0.13)$ & $2.06(0.11)$ & $2.56(0.30)$ & $\cdots$ \\
\hline
\end{tabular}




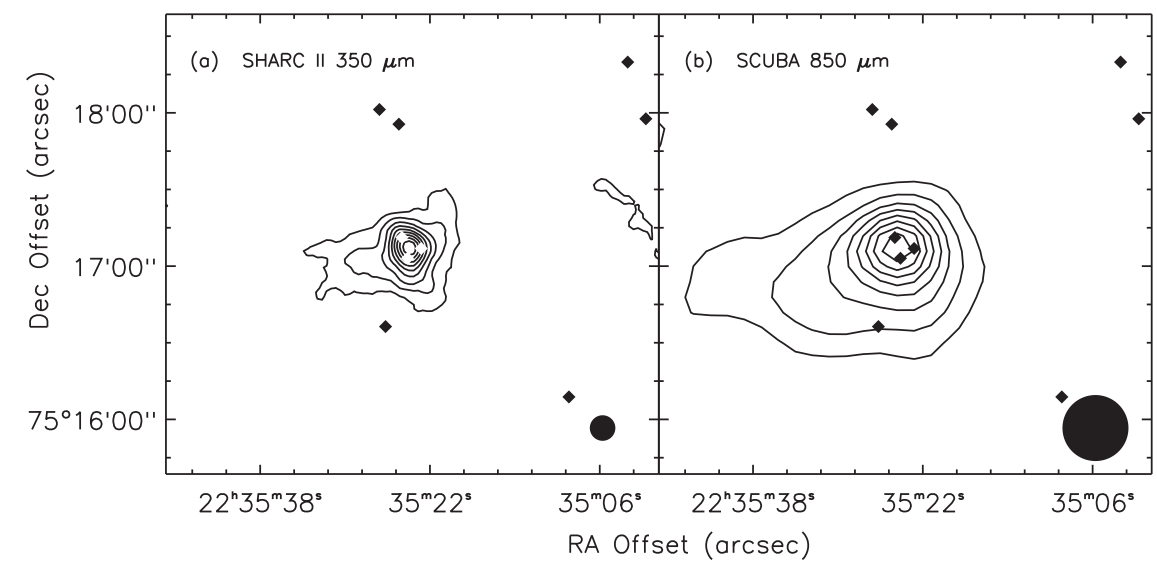

Figure 3. Dust continuum emission map toward L1251A. (a) Continuum emission at $350 \mu$ m obtained with SHARC-II at the CSO (Wu et al. 2007). Contour levels are 20, 40, 60, 80, and 100\% of the peak intensity, $5.40 \mathrm{Jy} \mathrm{beam}^{-1}$. (b) Continuum emission at $850 \mu \mathrm{m}$ obtained with SCUBA at the JCMT (Di Francesco et al. 2008). Contour levels are $20,40,60,80$, and $100 \%$ of the peak intensity, $1.20 \mathrm{Jy} \mathrm{beam}^{-1}$. The respective beam FWHM is presented in each panner with filled circle. YSO candidates are denoted by filled diamonds.

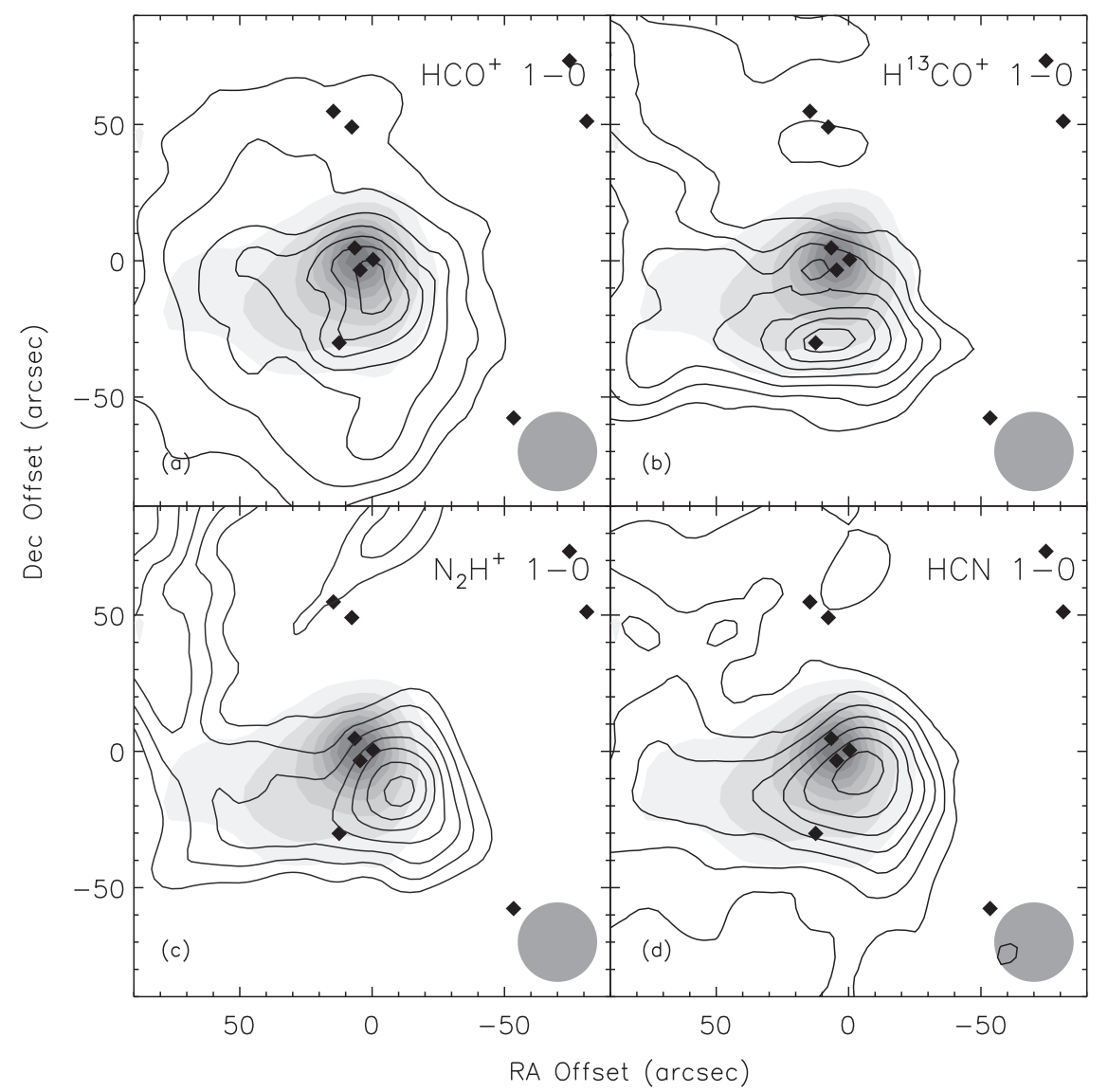

Figure 4. Integrated intensity maps of (a) $\mathrm{HCO}^{+} 1-0$, (b) $\mathrm{H}^{13} \mathrm{CO}^{+} 1-0$, (c) $\mathrm{N}_{2} \mathrm{H}^{+} 1-0$, and (d) $\mathrm{HCN} 1-0$ on top of the $850 \mu$ m map (gray scale). Contour intervals are $10 \%$ of the each peak intensity and range from 35 to $95 \%$. The gray scale levels are $20,40,60,80$, and $100 \%$ of the peak intensity, $1.20 \mathrm{Jy}^{2}$ beam ${ }^{-1}$. YSO candidates are denoted by filled diamonds. The filled circle at the bottom right of each panel denotes the respective beam FWHM.

calculated with the $850 \mu$ m integrated flux, $F_{\nu}$, using

$$
M_{\text {total }}=\frac{F_{\nu} d^{2}}{\kappa_{\nu} B_{\nu}\left(T_{\text {dust }}\right)}
$$

and assuming that all dust-continuum emission at $850 \mu \mathrm{m}$ arises from dust and is optically thin. Here, $d$ is the distance $\left(300 \pm 50 \mathrm{pc}\right.$; Kun \& Prusti 1993) and $B_{\nu}$ is the Planck function for a blackbody with a dust temperature of $T_{\text {dust }}$. To estimate the mass, we assumed $T_{\text {dust }}=20 \mathrm{~K}$ and adopted the mass opacity coefficient, $\kappa_{\lambda}=0.1(250 \mu \mathrm{m} / \lambda)^{\beta} \mathrm{cm}^{2} \mathrm{~g}^{-1}$ (Hildebrand 1983) with $\beta=1.8$ (Hatchell et al. 2013). This mass opacity assumes a gas-to-dust ratio of 100 , and the estimated mass represents the total (gas+dust) mass of the molecular core. Since $350 \mu \mathrm{m}$ is not in the Rayleigh-Jeans limit for this temperature regime, we derived the total mass using only the 
$850 \mu$ m flux. The derived total mass is $4.98 M_{\odot}$ with the integrated flux density $6.18 \mathrm{Jy}$. The aperture size used for the flux density is $60^{\prime \prime}$.

\subsubsection{Radio Single-dish Molecular Line Emission}

Figure 4 presents the integrated intensity maps of the $\mathrm{HCO}^{+}$ $1-0, \mathrm{H}^{13} \mathrm{CO}^{+} 1-0, \mathrm{~N}_{2} \mathrm{H}^{+} 1-0$, and $\mathrm{HCN} 1-0$, which all trace dense gas, made from the KVN single-dish observations. We integrated over the full widths of $\mathrm{HCO}^{+} 1-0$ and $\mathrm{H}^{13} \mathrm{CO}^{+} 1-0$, and all of the components of the hyperfine structures were integrated for the integrated intensity maps of $\mathrm{N}_{2} \mathrm{H}^{+} 1-0$ and $\mathrm{HCN} 1-0$. The molecular line integrated intensity extends in the $\mathrm{E}-\mathrm{W}$ direction in a manner similar to the distribution of the dust-continuum emission, except for $\mathrm{HCO}^{+} 1-0$ which may trace both infall and outflow (Figure 3). The integrated intensity peaks of these molecular lines are offset from the continuum peak position of L1251A, and relatively optically thin lines such as $\mathrm{H}^{13} \mathrm{CO}^{+} 1-0$ and $\mathrm{N}_{2} \mathrm{H}^{+} 1-0$ show bigger offsets. The integrated intensity peak of $\mathrm{H}^{13} \mathrm{CO}^{+} 1-0$ is $\sim 30^{\prime \prime}$ to the south of L1251A (Figure 4(b)) and is coincident with the position of IRS 14. The integrated intensity peak of $\mathrm{N}_{2} \mathrm{H}^{+}$ $1-0$ is $\sim 20^{\prime \prime}$ to the southwest of L1251A (Figure 4(c)). The integrated intensity peaks of relatively optically thick molecular emission such as $\mathrm{HCO}^{+} 1-0$ and $\mathrm{HCN} 1-0$ are slightly shifted to the southwest. The disagreements in position between the intensity peaks of single-dish dust-continuum emission and the molecular line integrated intensity peaks might indicate that the distribution of molecular gas around L1251A is complicated because of four sources existing within about $25^{\prime \prime}$ (i.e., $0.04 \mathrm{pc}$ ). For example, heating by these sources can evaporate $\mathrm{CO}$ from the dust grain surfaces, which is a mother molecule of $\mathrm{HCO}^{+}$but a destroyer of $\mathrm{N}_{2} \mathrm{H}^{+}$.

Figure 5 shows the line profiles of the above molecular lines at the center position (IRS 1 ) as well as that of $\mathrm{H}_{2} \mathrm{CO} 2_{12}-1_{11}$. The spectrum of $\mathrm{HCO}^{+} 1-0$ toward L1251A shows a strong self-absorption dip with a classic blue asymmetric line profile, which was believed to be an infall indicator (Zhou et al. 1993). These dips imply true self-absorption when the velocity of the line peak of optically thin tracers such as $\mathrm{H}^{13} \mathrm{CO}^{+} 1-0, \mathrm{H}_{2} \mathrm{CO}$ $2{ }_{12}-1_{11}$, and $\mathrm{N}_{2} \mathrm{H}^{+} 1-0$ coincides with the velocity of the dip; although the centroid velocities of optically thin tracers such as $\mathrm{H}^{13} \mathrm{CO}^{+} 1-0$ and $\mathrm{H}_{2} \mathrm{CO} 2_{12}-1_{11}$ are slightly blueshifted, they peak at the velocity of the self-absorption dip. The Gaussian fit FWHMs line widths of $\mathrm{H}^{13} \mathrm{CO}^{+} 1-0$ and $\mathrm{H}_{2} \mathrm{CO} 2_{12}-1_{11}$ are 1.3 and $1.9 \mathrm{~km} \mathrm{~s}^{-1}$, respectively. From simultaneous hyperfine structure fitting, the FWHMs of $\mathrm{N}_{2} \mathrm{H}^{+} 1-0$ and $\mathrm{HCN} 1-0$ are 0.6 and $1.04 \mathrm{~km} \mathrm{~s}^{-1}$, respectively. Therefore, $\mathrm{N}_{2} \mathrm{H}^{+} 1-0$ seems to trace the most quiescent gas component. In addition, the profile of the $F=2-1$ hyperfine component of HCN 1-0 line, which also has been suggested as an infall tracer, is similar to that of $\mathrm{HCO}^{+} 1-0$.

The line profile of $\mathrm{HCO}^{+} 1-0$ is superimposed by two (broad and narrow) components and the narrow component shows the self-absorption feature. The broad component $\left(v_{\text {cen }} \sim-4.8\right.$ $\left.\mathrm{km} \mathrm{s}^{-1}, \Delta v \sim 2.9 \mathrm{~km} \mathrm{~s}^{-1}\right)$ is seen in all positions across the observed map while the narrow component $\left(v_{\text {cen }} \sim-5.3\right.$ $\mathrm{km} \mathrm{s}^{-1}, \Delta v \sim 0.8 \mathrm{~km} \mathrm{~s}^{-1}$ ) is detected only around L1251A. The integrated intensity map of the broad component (not shown) shows a distribution similar to the $\mathrm{HCO}^{+} 1-0$ outflow (see Section 4.3 below), suggesting that the origin of the broad component may be outflows from L1251A. The integrated intensity map of the $\mathrm{HCO}^{+} 1-0$ narrow component and that of the isolated component of $\mathrm{N}_{2} \mathrm{H}^{+} 1-0$ show similar distributions, although the integrated intensity peaks of the two lines do not coincide (Figure 6). The similarity suggests that the narrow component of $\mathrm{HCO}^{+} 1-0$ may trace the dense and relatively quiescent molecular gas around L1251A like the other molecular lines (except for CO 1-0).

\subsubsection{Radio Single-dish Water Maser Emission}

$\mathrm{H}_{2} \mathrm{O}$ maser emission at $22 \mathrm{GHz}$ was monitored toward L1251A to detect variations in the systemic velocity and intensity in time. In Figure 7, multi-epoch results are presented. Maser components of different velocity or intensity have been observed at different epochs. Five redshifted maser components can be identified at $-2.4,0,2,3.2$, and $153 \mathrm{~km} \mathrm{~s}^{-1}$. The $-2.4 \mathrm{~km} \mathrm{~s}^{-1}$ component was presented in 2013 February, slightly weakened in 2013 March, and became very strong in 2013 May. The $0 \mathrm{~km} \mathrm{~s}^{-1}$ component was first detected in 2012 December and became stronger in 2013 January but weakened in 2013 May. The $3.2 \mathrm{~km} \mathrm{~s}^{-1}$ velocity component and the very high velocity component, $153 \mathrm{~km} \mathrm{~s}^{-1}$, were also detected in 2013 February (Figure 8). The integrated intensity peak of the $\mathrm{H}_{2} \mathrm{O}$ maser emission (not shown) is located in L1251A but the beam FWHM is not small enough to identify the specific location of the water maser source.

The isotropic luminosity of the $\mathrm{H}_{2} \mathrm{O}$ maser can be estimated by the integrated intensities of the observed emission using the following equation (Bae et al. 2011):

$$
L_{\mathrm{H}_{2} \mathrm{O}}=4 \pi d^{2} \frac{v}{c} \int F_{\nu} d v .
$$

Here, $d$ is the distance $(300 \pm 50 \mathrm{pc}$; Kun \& Prusti 1993) and $\nu$ is the observed frequency. When multiple lines are present, the sum of all the individual lines are used to calculate the luminosity. The derived $\mathrm{H}_{2} \mathrm{O}$ maser luminosities are presented in Table 4 .

\subsubsection{Radio Interferometer Continuum Emission}

The $1.3 \mathrm{~mm}$ continuum image obtained with SMA toward L1251A is presented in Figure 9. Three individual condensations were detected. For the $1.3 \mathrm{~mm}$ continuum sources, we will follow the nomenclature of Reipurth et al. (2004). Although the peak positions of submillimeter sources are not exactly coincident with the positions of IR sources, VLA 6 and VLA 7 are the same sources as IRS 1 and IRS 3, respectively. However, VLA 10 is separated from IRS 2 by about $2^{\prime \prime}$. The positions of IRS 2 and the 2MASS source are consistent. Therefore, VLA 10 and IRS 2 are tracing two different sources. The dust-continuum emission related to VLA 6 and VLA 7 was first detected by Meehan et al. (1998, their VLA A and VLA B) at $3.6 \mathrm{~cm}$ and next by Beltrán et al. (2001) at 3.6 and $6 \mathrm{~cm}$. Reipurth et al. (2004), however, first detected the continuum condensation associated with VLA 10 at $3.6 \mathrm{~cm}$, and they mentioned that VLA 10 is a radio jet source at a position angle of about $53^{\circ}$, indicating that VLA 10 is another protostellar member in L1251A, although no corresponding IR source has been detected. Reipurth et al. (2004) also mentioned that VLA 7 is a radio jet source at a position angle of about $126^{\circ}$.

Although VLA 6 and VLA 10 are connected at low intensity levels, three sources are resolved in our $1.3 \mathrm{~mm}$ continuum map. To estimate their masses, we used $T_{\text {dust }}=30 \mathrm{~K}$ (Jørgensen et al. 2007) and adopted the dust mass opacity coefficient, $\quad \kappa_{\lambda}=0.02(1 \mathrm{~mm} / \lambda)^{\beta} \mathrm{cm}^{2} \mathrm{~g}^{-1} \quad$ (Beckwith \& 

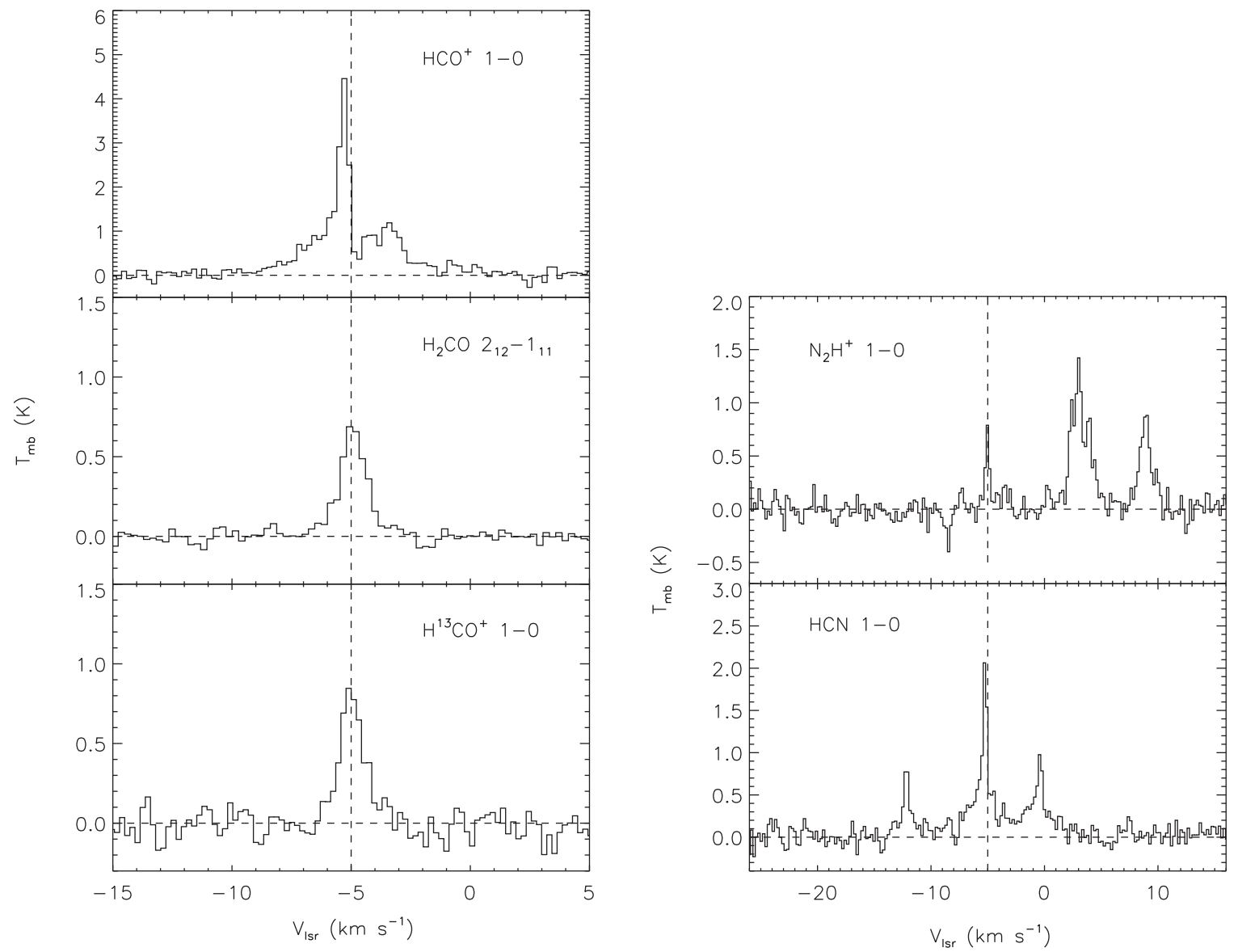

Figure 5. Spectra of all of the molecular transitions observed with KVN at the center position of the single-dish molecular line map. The horizontal dashed lines and the vertical dashed lines represent the zero baseline and the systemic velocity $\left(-5 \mathrm{~km} \mathrm{~s}^{-1}\right)$ of L1251-C, respectively.

(a) $\mathrm{HCO}^{+} 1-0$ narrow component

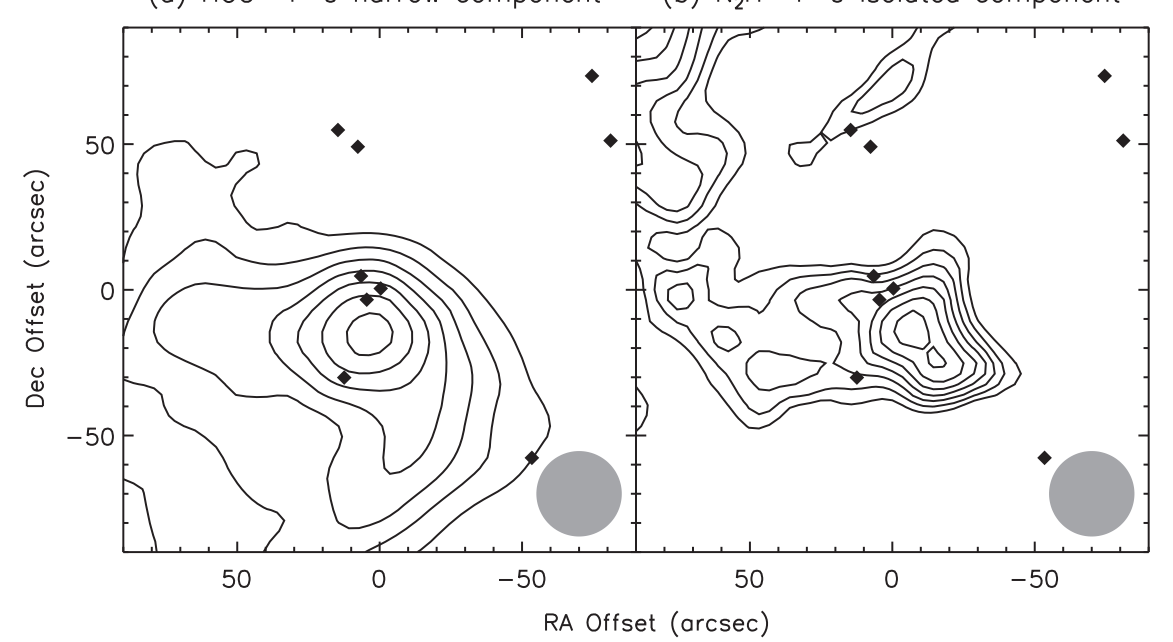

Figure 6. Integrated intensity maps of (a) the $-5.3 \mathrm{~km} \mathrm{~s}^{-1}$ (narrow) component of $\mathrm{HCO}^{+} 1-0$ and (b) the $-5 \mathrm{~km} \mathrm{~s}^{-1}$ (isolated) component of $\mathrm{N}_{2} \mathrm{H}^{+} 1-0$. YSO candidates are denoted by filled diamonds. Contour intervals are $10 \%$ of each peak intensity and range from 35 to $95 \%$. The filled circle at the bottom right of each panel denotes the respective beam FWHM.

Sargent 1991), which is normalized for flat sources $(\beta=1)$. The masses of VLA 6, VLA 7, and VLA 10 are presented in Table 5. The total mass derived from the SCUBA $850 \mu$ m continuum map is roughly 55 times larger than the sum of the masses derived from the SMA $1.3 \mathrm{~mm}$ continuum sources because the single-dish flux includes extended emission around all four sources.

\subsubsection{Radio Interferometer Molecular Line Emission}

Line profiles of $\mathrm{CO} 2-1$ and ${ }^{13} \mathrm{CO} 2-1$, observed with the SMA at the position of each submillimeter source, are presented in Figure 10. The channel widths for CO 2-1 and ${ }^{13} \mathrm{CO} 2-1$ are 1.09 and $0.23 \mathrm{~km} \mathrm{~s}^{-1}$, respectively. Among the three submillimeter sources, the strongest peak brightness 


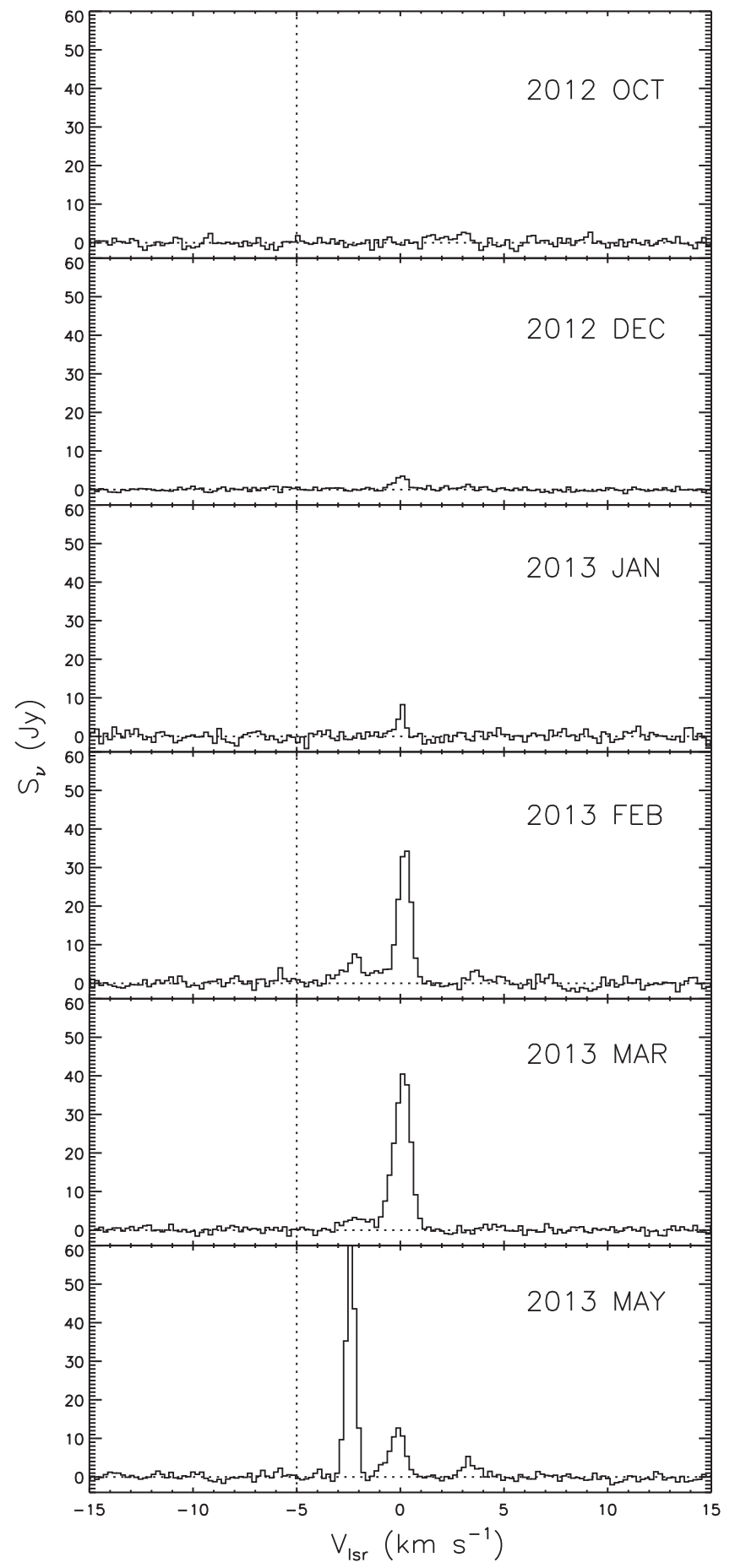

Figure 7. Spectra of the $22 \mathrm{GHz} \mathrm{H}_{2} \mathrm{O}$ maser line toward L1251A. The observation dates are labeled. The horizontal dotted line and the vertical dotted line represent the zero baseline and the systemic velocity $\left(-5 \mathrm{~km} \mathrm{~s}^{-1}\right)$ of L1251-C, respectively.

temperature of $\mathrm{CO} 2-1(24.75 \mathrm{~K})$ is seen at VLA 6 , while the peak brightness temperatures of ${ }^{13} \mathrm{CO} 2-1(\sim 7 \mathrm{~K})$ are similar toward all three sources. The centroid velocity of ${ }^{13} \mathrm{CO} 2-1$ is shifted from red to blue in the order of VLA 6, VLA 10, and VLA 7. There are channels without emission in ${ }^{13} \mathrm{CO} 2-1$ at $v_{\mathrm{lsr}}$ $=-4.94$ and $-3.84 \mathrm{~km} \mathrm{~s}^{-1}$. The two dips of ${ }^{13} \mathrm{CO} 2-1$ cannot be resolved with the $\sim 1 \mathrm{~km} \mathrm{~s}^{-1}$ velocity resolution of the $\mathrm{CO}$ $2-1$ data. Moreover, $\mathrm{CO} 2-1$ emission is missing in the velocity range $v_{\mathrm{lsr}}=-6$ to $-4 \mathrm{~km} \mathrm{~s}^{-1}$. At these velocities, most emission

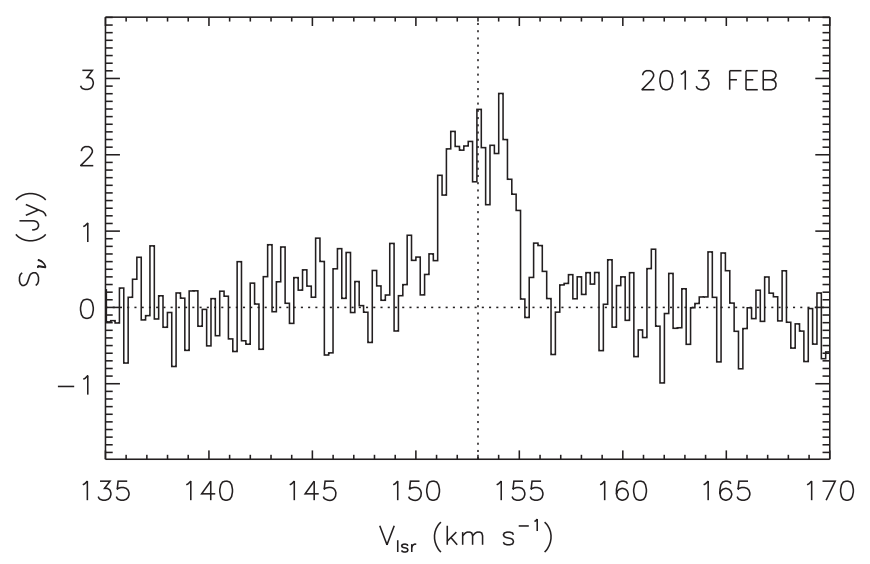

Figure 8. Highest velocity component of $22 \mathrm{GHz} \mathrm{H}_{2} \mathrm{O}$ maser line toward L1251A. This high-velocity component was detected only on 2013 February. The horizontal dotted line and the vertical dotted line represent the zero baseline and the central velocity of the highest-velocity component, respectively.

Table 4

The Isotropic Luminosity of the $\mathrm{H}_{2} \mathrm{O}$ Maser

\begin{tabular}{lc}
\hline \hline Observing Date & $\begin{array}{c}L_{\mathrm{H}_{2} \mathrm{O}} \\
\left(L_{\odot}\right)\end{array}$ \\
\hline $2012 \mathrm{Dec}$ & $9.46 \mathrm{E}-09$ \\
$2013 \mathrm{Jan}$ & $5.55 \mathrm{E}-09$ \\
$2013 \mathrm{Feb}$ & $7.41 \mathrm{E}-08$ \\
2013 Mar & $9.59 \mathrm{E}-08$ \\
2013 May & $2.01 \mathrm{E}-07$ \\
\hline
\end{tabular}

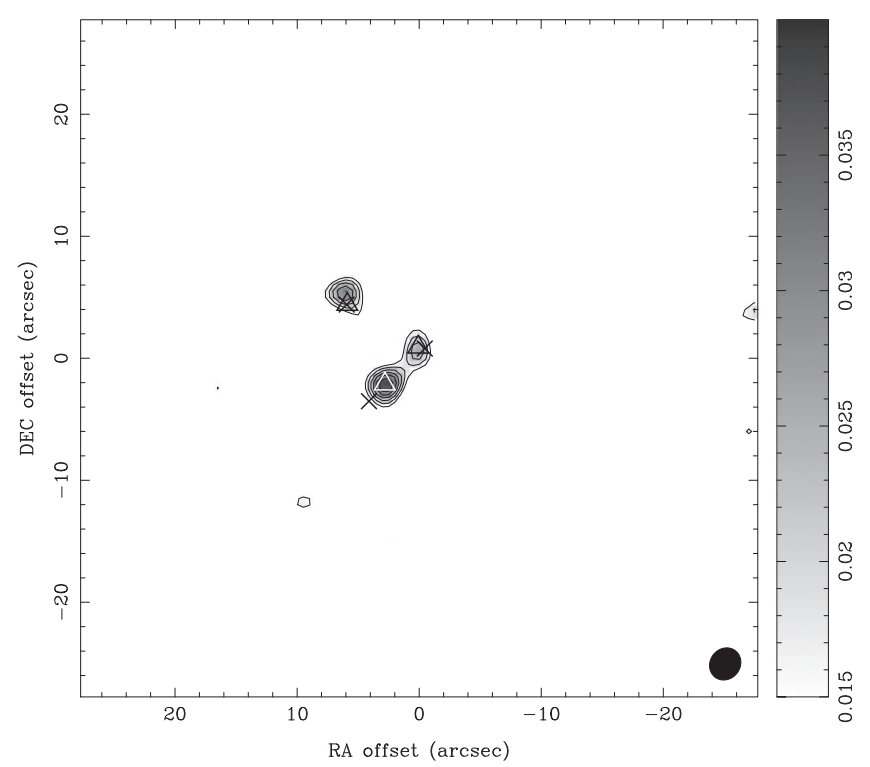

Figure 9. $1.3 \mathrm{~mm}$ dust continuum emission map toward L1251A obtained with SMA. Contour levels are $40,50,60,70,80$, and $90 \%$ of the peak intensity, $0.038 \mathrm{Jy} \mathrm{beam}^{-1}$, and the wedge indicates the continuum intensity scale in units of Jy beam ${ }^{-1}$. The synthesized beam FWHM is presented at the bottom right corner. The rms noise level $1 \sigma$ is $4.7 \mathrm{mJy}_{\text {beam }}{ }^{-1}$. The positions of IR sources in L1251A and the position of VLA $3.6 \mathrm{~cm}$ continuum sources (Reipurth et al. 2004) are denoted by Xs and triangles, respectively. The size of this presented map is the same as the primary beam size at this frequency $\left(\sim 55^{\prime \prime}\right)$. 
Table 5

Parameters of the SMA $1.3 \mathrm{~mm}$ Dust Continuum Condensations Associated with L1251A

\begin{tabular}{|c|c|c|c|c|c|c|}
\hline ID & 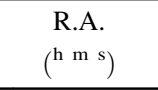 & $\begin{array}{l}\text { Decl. } \\
\left({ }^{\circ}, \prime \prime\right)\end{array}$ & $\begin{array}{c}\text { Peak Flux } \\
\left(\text { mJy beam }^{-1}\right)\end{array}$ & $\begin{array}{l}\text { Integrated Flux Density } \\
\qquad(\mathrm{mJy})\end{array}$ & $\begin{array}{c}\text { Source Size } \\
\left({ }^{\prime \prime} \times^{\prime \prime}\right)\left(\text { P.A. }^{\circ}\right)\end{array}$ & $\begin{array}{l}\text { Mass } \\
\left(M_{\odot}\right)\end{array}$ \\
\hline VLA 6 (IRS 1) & $22: 35: 23.45$ & $+75: 17: 07.73$ & $24.20(0.001)$ & 35.23 & $3.9 \times 2.5(-0.9)$ & 0.024 \\
\hline VLA 7 (IRS 3) & $22: 35: 24.98$ & $+75: 17: 12.17$ & $31.83(0.003)$ & 40.53 & $3.3 \times 2.7(55.5)$ & 0.028 \\
\hline VLA 10 & $22: 35: 24.11$ & $+75: 17: 04.81$ & $38.36(0.004)$ & 53.12 & $3.3 \times 2.8(-30.6)$ & 0.037 \\
\hline
\end{tabular}

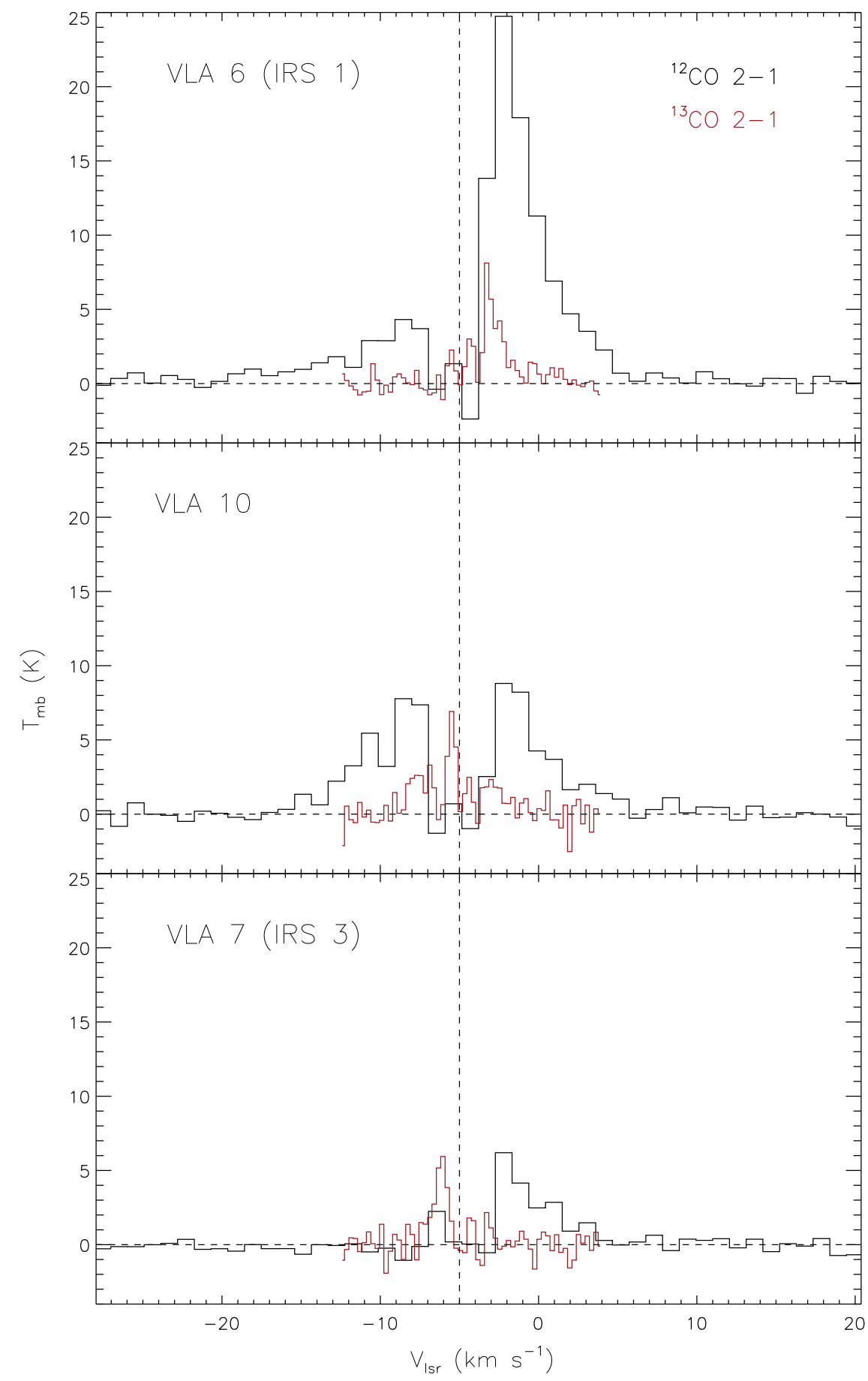

Figure 10. Spectra of $\mathrm{CO} 2-1$ (black) and ${ }^{13} \mathrm{CO} 2-1$ (red) observed with SMA toward the three SMA 1.3 mm continuum sources, VLA 6, VLA 10, and VLA 7, from top to bottom. The black dotted vertical lines indicate the systemic velocity of L1251-C, $-5.0 \mathrm{~km} \mathrm{~s}^{-1}$, obtained from single-dish observations. 


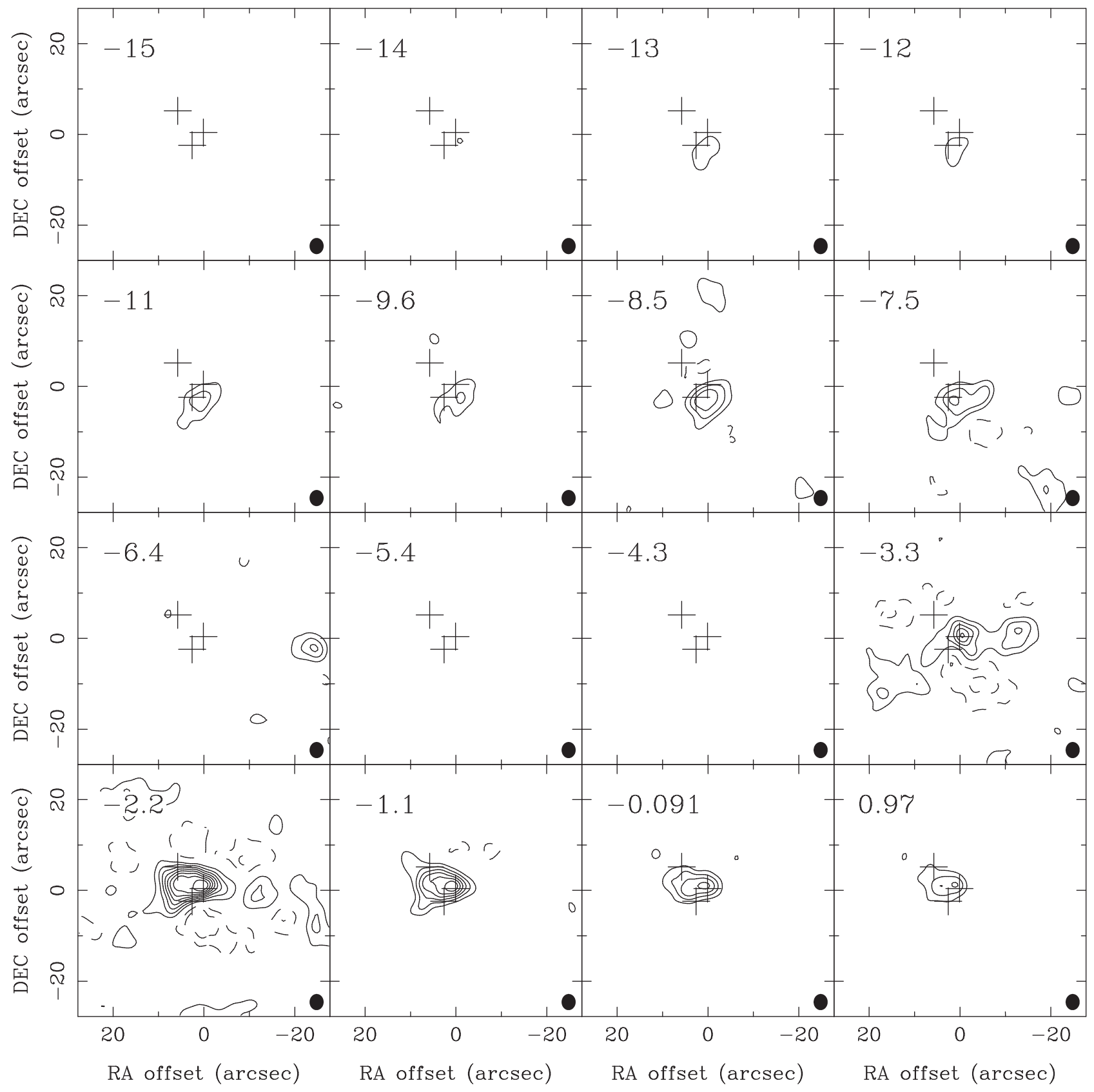

Figure 11. Channel map of CO 2-1 observed with the SMA. Respective velocities in $\mathrm{km} \mathrm{s}^{-1}$ are indicated in the upper left of each panel. The contours start from $5 \sigma$ and increase in steps of $5 \sigma$, where $1 \sigma$ is $0.268 \mathrm{Jy} \mathrm{beam}^{-1}$. Dashed contours denote negative intensities with the same steps as positive contours. Crosses denote the SMA $1.3 \mathrm{~mm}$ continuum source positions (VLA 6, VLA 7, and VLA 10). The synthesized beam FWHM is shown by the black ellipse.

from the larger-scale structure in that velocity range has likely been resolved out.

In Figure 11, the velocity channel map of the CO 2-1 line is presented. The $\mathrm{CO} 2-1$ emission is in the velocity range -14.9 to $5.2 \mathrm{~km} \mathrm{~s}^{-1}$. In the channel map, the CO $2-1$ emission is missing in the -5.4 and $-4.3 \mathrm{~km} \mathrm{~s}^{-1}$ channels. Moreover, the strong negative contours are shown near bright emission caused by the missing flux effect at -3.3 and $-2.2 \mathrm{~km} \mathrm{~s}^{-1}$. A blueshifted component related to VLA 6 or VLA 10 is seen to extend toward the southwest of L1251A. A strong redshifted component is located at the position of VLA 6, extending in the northeast direction. Although it is hard to distinguish apparent bipolar outflow structures, we infer that the blue- and redshifted components are from outflowing gas because the velocity offsets from the systemic velocity are $\geqslant 2.5 \mathrm{~km} \mathrm{~s}^{-1}$. We will further discuss outflows in Section 4.3 below. Figure 12 shows the CO 1-0 integrated intensity across L1251A overlaid on the $1.3 \mathrm{~mm}$ continuum emission map for reference. The integrated intensity map of $\mathrm{CO} 1-0$ shows $\mathrm{E}-\mathrm{W}$ elongation and the intensity peak is located at the position of VLA 6 .

Figure 13 shows the velocity channel map of the ${ }^{13} \mathrm{CO}$ 2-1 line. Every four channels are integrated for ${ }^{13} \mathrm{CO} 2-1$ to compare with the CO 2-1 channel map. The redshifted emission at the position of VLA 6 is shown at $-3.1 \mathrm{~km} \mathrm{~s}^{-1}$, while the blueshifted emission at the position of VLA 10 and VLA 7 is shown at -5.4 and $-6.5 \mathrm{~km} \mathrm{~s}^{-1}$, respectively. The blueshifted emission at $-5.4 \mathrm{~km} \mathrm{~s}^{-1}$ is slightly extended to the southwest and the blueshifted emission at $-6.5 \mathrm{~km} \mathrm{~s}^{-1}$ is 


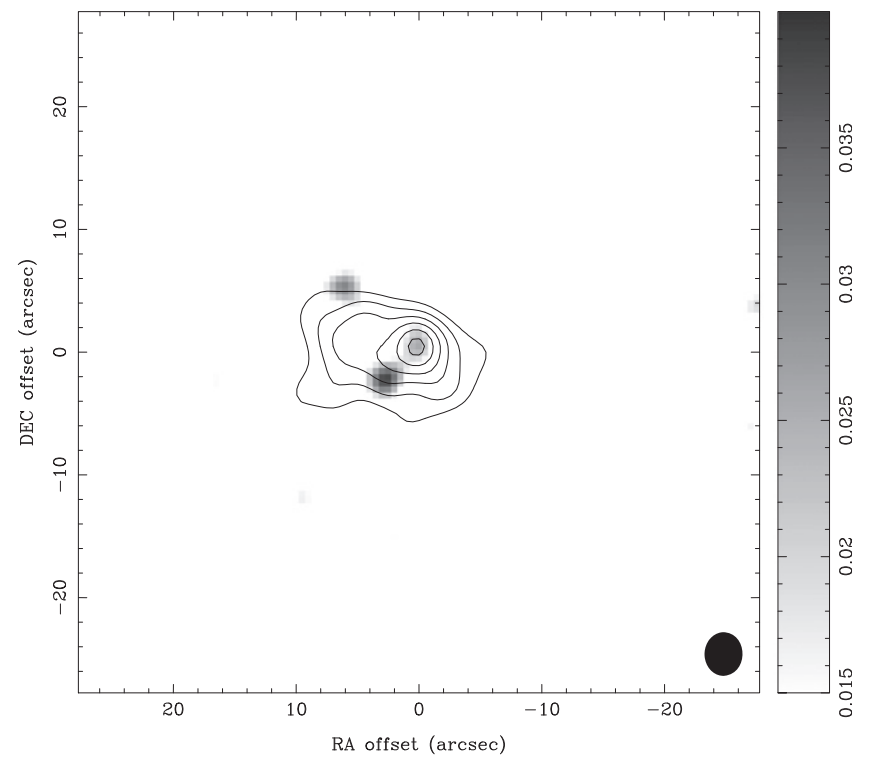

Figure 12. Integrated intensity map of $\mathrm{CO} 2-1$ overlaid on the $1.3 \mathrm{~mm}$ continuum emission (gray scale). The contours represent 30, 45, 60, 75, 80, and $95 \%$ of the peak intensity of $57.3 \mathrm{Jy} \mathrm{beam}^{-1} \mathrm{~km} \mathrm{~s}^{-1}$ and the wedge indicates the continuum emission scale from 0.015 to $0.040 \mathrm{Jy}^{\text {beam }}{ }^{-1}$. The synthesized beam FWHM is shown by the black ellipse. The map covers the primary beam size at this frequency $\left(\sim 55^{\prime \prime}\right)$.

extended to the northeast. At $-5.4 \mathrm{~km} \mathrm{~s}^{-1}$, there is another blob to the east of VLA 10 which can be discerned in the integrated intensity map of ${ }^{13} \mathrm{CO} 2-1$. In Figure 14, the integrated intensity map of ${ }^{13} \mathrm{CO}$ 2-1 across L1251A is presented and also overlaid onto the $1.3 \mathrm{~mm}$ continuum emission map for reference. Each continuum source seems to be associated with a ${ }^{13} \mathrm{CO} \quad 2-1$ integrated intensity peak, although those peaks are not completely distinct. The whole emission structure is extended along the NE-SW direction.

Most of the emission of the blue- and redshifted components of ${ }^{13} \mathrm{CO} 2-1$ is seen in the velocity range where the $\mathrm{CO}$ 2-1 emission appears to have been resolved out. In addition, the blue- and redshifted components of ${ }^{13} \mathrm{CO} 2-1$ have low velocities (within $v_{\mathrm{lsr}} \pm 3 \mathrm{~km} \mathrm{~s}^{-1}$ ) compared to that of $\mathrm{CO}$ $2-1$ (within $v_{\mathrm{lsr}} \pm 9 \mathrm{~km} \mathrm{~s}^{-1}$ ). Therefore, the features of ${ }^{13} \mathrm{CO}$ $2-1$ seem to trace the innermost part of the envelopes around each submillimeter source rather than outflows.

\section{ANALYSIS}

\subsection{YSO Classification}

Except for IRS 1, IRS 2, and IRS 3, identified by the HDR observations, the IR spectral indexes $\alpha$ of 16 YSO candidates are listed in the c2d YSOc catalog. The $\alpha$ for IRS 1, IRS 2, and IRS 3 are calculated in this work. All photometries between 2 and $24 \mu \mathrm{m}$ in the c2d catalog are used to determine the IR spectral index $\alpha$ (Evans et al. 2009):

$$
\alpha=\frac{d \log (\lambda S(\lambda))}{d \log (\lambda)} .
$$

Here, $\lambda$ is the wavelength and $S(\lambda)$ is the flux density at that wavelength. Following the criteria of Evans et al. (2009; i.e., Class I for $0.3 \leqslant \alpha$, Flat $-0.3 \leqslant \alpha<0.3$, Class II for -1.6 $\leqslant \alpha<-0.3$, and Class III for $\alpha<-1.6$ ), IRS 1, 11, and 16 are
Class I candidates, IRS 3, 7, and 17 are Flat class candidates, and all others are Class II candidates. Table 6 lists the IR spectral index determined for each source. We calculated the IR spectral indices of the newly added YSOs, also listed in Table 6. We also applied two other classification criteria (the color-color diagram and $T_{\text {bol }}$ ) to all 19 YSOs. The photometric data of all YSOs are presented in Table 3 from the Spitzer observations. Figure 15 shows a [3.6]-[4.5] versus [5.6]-[8.0] color-color diagram used to classify the evolutionary stages of YSO candidates, including all identified sources in Figure 2. We adopted the same criteria used in Lee et al. (2006), which is basically based on the models of Allen et al. (2004). Two sources, IRS 1 and 14, are identified as Class 0/I candidates in color-color diagrams. IRS 11 and 17 are located at the colorcolor boundaries between Class $0 / I$ and Class II. The IR spectral indices of these sources, however, indicate that IRS 11 is in Class I and IRS 17 is in the Flat class. IRS 4 is located close to the boundary of the Class III criteria, but its IR spectral index of IRS 4 is in the range for Class II.

In Table 6 , derived bolometric luminosities $\left(L_{\mathrm{bol}}\right)$ and bolometric temperatures $\left(T_{\text {bol }}\right)$ are listed. If the classification by Myers \& Ladd (1993; i.e., Class 0 for $T_{\text {bol }}<70 \mathrm{~K}$, Class I for $70 \mathrm{~K} \leqslant T_{\text {bol }} \leqslant 650 \mathrm{~K}$, and Class II for $650 \mathrm{~K}<T_{\text {bol }} \leqslant 2800 \mathrm{~K}$ ) is used, then IRS 1, 11, and 16 are identified as Class I, and the other 16 YSOs are identified as Class II. The evolutionary stages of most sources identified by $T_{\text {bol }}$ agree with those provided by the IRAC color-color diagram. Note, however, that IRS 14 is identified as an embedded source by the colorcolor diagram but as Class II by $T_{\text {bol }}$ and $\alpha$. It is more likely in Class II, but since it is spatially located at the $\mathrm{H}^{13} \mathrm{CO}^{+}$ $1-0$ emission peak it may be Class $0 / I$ instead. Furthermore, IRS 11, which is located at the boundaries of Class $0 / \mathrm{I}$ and Class II in the IRAC color-color diagram, is identified as Class I by $T_{\text {bol }}$ (IRS 11 has a very low luminosity). In addition, IRS 4 is identified as Class II source by $T_{\text {bol }}$. Finally, IRS 16 is identified as a Class I source with the lowest $T_{\text {bol }}$ among 19 YSO candidates.

IRS 11 and IRS 16 are identified as Class I YSOs with luminosities smaller than $0.1 L_{\odot}$, suggesting that these sources are candidates of Very Low Luminosity Objects (e.g., Young et al. 2004; Bourke et al. 2005; Dunham et al. 2006). However, it cannot be ruled out that these two sources may be background galactic sources. Evans et al. (2007) defined the probability of being a background galactic source, Log(ProbGalc), and galaxy candidates have Log(Prob_Galc) $\geqq-1.47$. For IRS 11 and IRS 16, the probabilities are -2.17 and -1.58 , respectively.

Spectral energy distributions (SEDs) for the YSOs in L1251$\mathrm{C}$ are presented in Figure 16. The SEDs include 2MASS, IRAC, and MIPS bands data. IRS 1 is the brightest source at near- and mid-infrared wavelengths, except in the IRAC 1 band $(3.6 \mu \mathrm{m})$.

\subsection{L1251A and the Common Envelope}

Including the radio continuum source VLA 10, there are four members in L1251A. The mean projected separation between each companion is about 1220 AU. Looney et al. (2000) classified multiple systems by their morphologies into three groups: separate envelope systems, common envelope systems, and common disk systems. The typical separation of common 


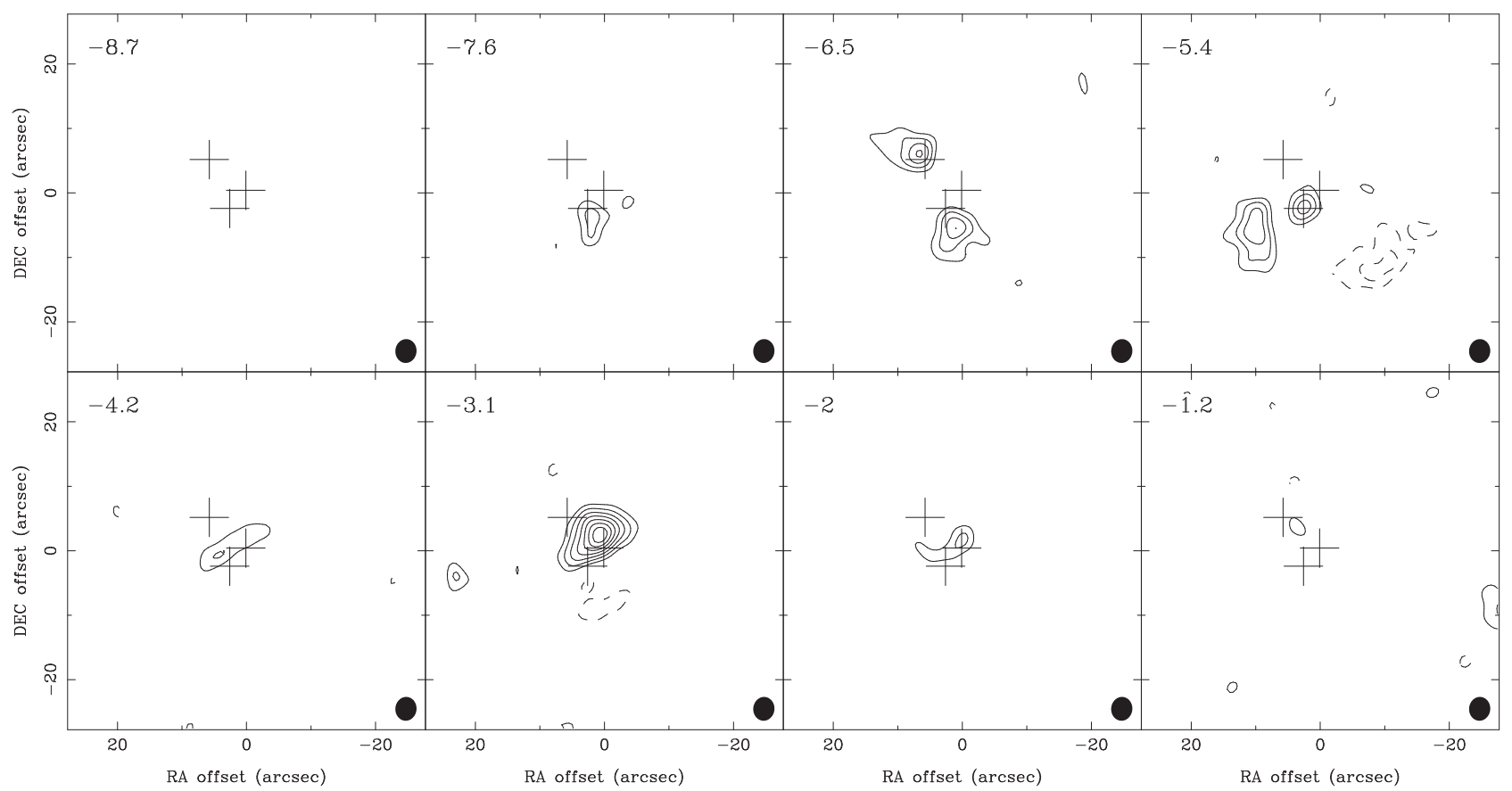

Figure 13. Channel map of ${ }^{13} \mathrm{CO} 2-1$ observed with the SMA. Respective velocities in $\mathrm{km} \mathrm{s}^{-1}$ are indicated in the upper left of each panel. The contours start from $2 \sigma$ and increase in steps of $1 \sigma$, where $1 \sigma=0.404 \mathrm{Jy} \mathrm{beam}^{-1}$. Dashed contours show the negative intensities with same steps of positive contours. The synthesized beam FWHM is shown by the black ellipse.

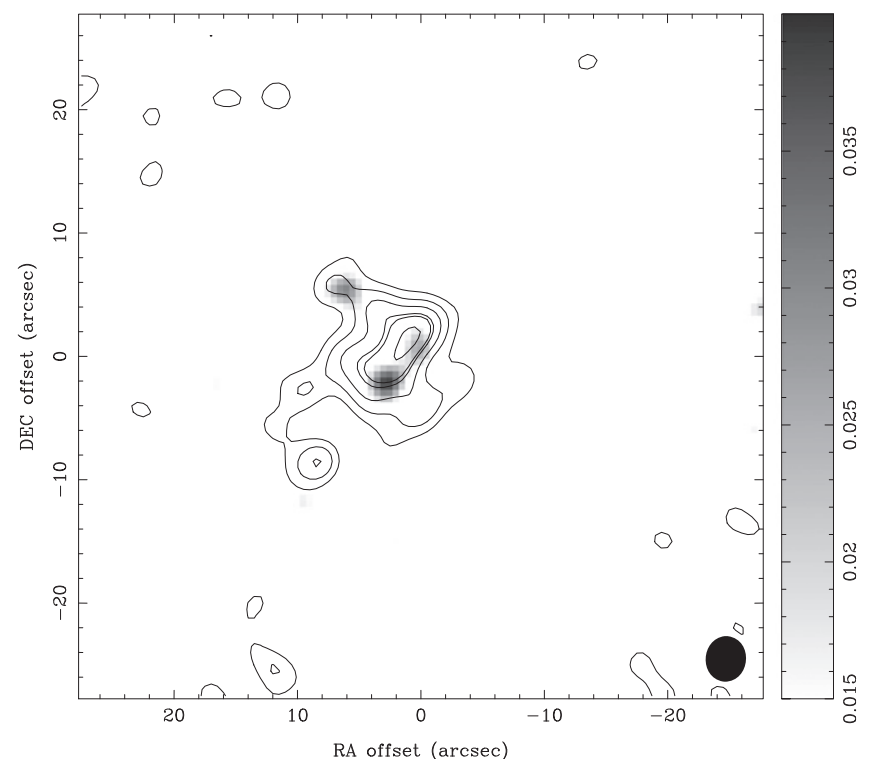

Figure 14. Integrated intensity map of ${ }^{13} \mathrm{CO} 2-1$ overlaid onto the $1.3 \mathrm{~mm}$ continuum (gray scale). The contours represent $25,40,55,70,85$, and $100 \%$ of the peak intensity of $7.75 \mathrm{Jy}_{\text {beam }}^{-1} \mathrm{~km} \mathrm{~s}^{-1}$ and the wedge indicates the continuum emission scale from 0.015 to $0.040 \mathrm{Jy}_{\text {beam }}{ }^{-1}$. The synthesized beam FWHM is shown by the black ellipse.

envelope systems ranges from 150 to $3000 \mathrm{AU}$. The projected distances among the members of this system suggest that L1251A is a common envelope system with one primary core of gravitational concentration.

\subsection{Molecular Outflows}

In L1251-C, an extended bipolar CO 1-0 outflow driven by IRAS $22343+7501$ was reported by Sato \& Fukui (1989). An optical jet was also observed (Balazs et al. 1992) with the same

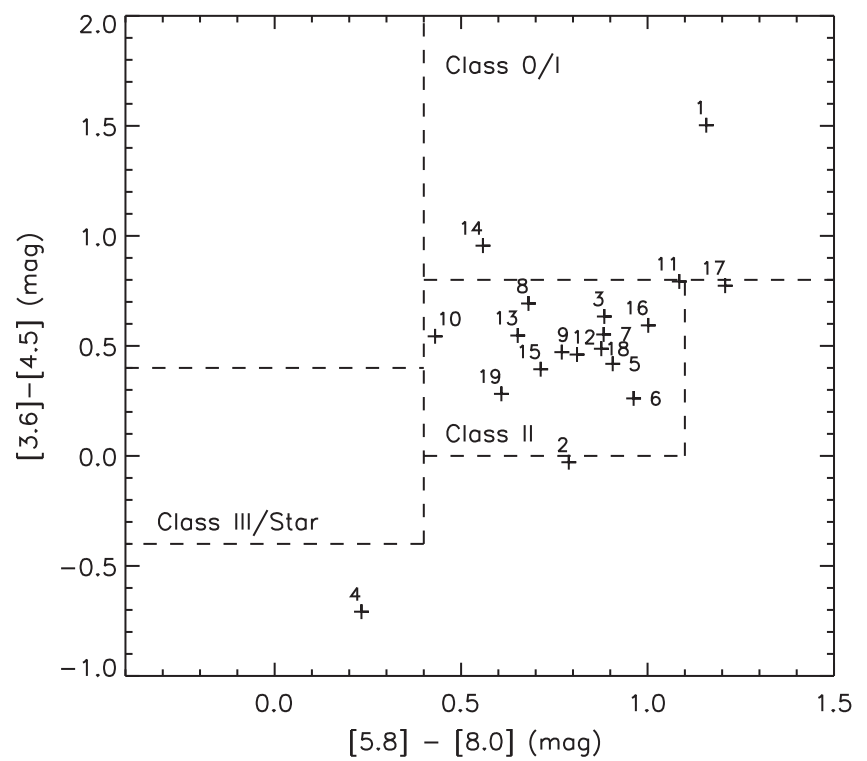

Figure 15. [3.6]-[4.5] vs. [5.6]-[8.0] color-color diagram for L1251-C YSO candidates in the IRAC bands. Source numbers are labeled. The dashed lines describe the approximate criteria for Class I, Class II, and Class III sources by Lee et al. (2006) based on Allen et al. (2004).

axis (NE-SW) as the extended CO 1-0 outflow. The outflow map of Schwartz et al. (1988), however, shows a bipolar structure with an unclear outflow axis in a small region around L1251A.

For this paper, the velocity ranges for blue- and redshifted outflow wings were defined as follows. The outer velocities of the ranges are determined for each position where the emission is greater than the respective $1 \sigma \mathrm{rms}$ noise level. Spectra at outflow-free positions, chosen by eye, are used to define the 
Table 6

Bolometric Luminosity, Bolometric Temperature, and IR Spectral Indices of YSOs in L1251-C

\begin{tabular}{|c|c|c|c|}
\hline Sources & $\begin{array}{l}L_{\text {bol }} \\
\left(L_{\odot}\right) \\
\end{array}$ & $\begin{array}{c}T_{\text {bol }}(\text { Class }) \\
(\mathrm{K})\end{array}$ & Spectral Index $\alpha$ (Class) \\
\hline 1 & 3.2 & $622(\mathrm{I})$ & $2.94(\mathrm{I})$ \\
\hline 2 & 0.54 & 1180 (II) & -0.72 (II) \\
\hline 3 & 2.4 & 1041 (II) & -0.12 (Flat) \\
\hline 4 & 3.1 & 1074 (II) & $-0.51(\mathrm{II})$ \\
\hline 5 & 0.25 & 813 (II) & -0.38 (II) \\
\hline 6 & 0.085 & 1440 (II) & $-0.84(\mathrm{II})$ \\
\hline 7 & 0.17 & 747 (II) & -0.29 (Flat) \\
\hline 8 & 0.18 & 910 (II) & -0.59 (II) \\
\hline 9 & 0.12 & 960 (II) & -0.54 (II) \\
\hline 10 & 0.042 & 1209 (II) & $-1.02(\mathrm{II})$ \\
\hline 11 & 0.015 & $367(\mathrm{I})$ & $0.35(\mathrm{I})$ \\
\hline 12 & 0.47 & 1321 (II) & $-0.84($ II $)$ \\
\hline 13 & 0.63 & 1422 (II) & $-1.05(\mathrm{II})$ \\
\hline 14 & 0.16 & 713 (II) & $-0.34(\mathrm{II})$ \\
\hline 15 & 0.24 & 1441 (II) & -0.91 (II) \\
\hline 16 & 0.004 & $174(\mathrm{I})$ & $1.20(\mathrm{I})$ \\
\hline 17 & 0.80 & 763 (II) & 0.10 (Flat) \\
\hline 18 & 0.35 & 1505 (II) & -0.49 (II) \\
\hline 19 & 0.014 & 1537 (II) & $-1.13(\mathrm{II})$ \\
\hline
\end{tabular}

inner velocities of the ranges as equal to the outermost velocities.

The averaged outflow-free spectra of $\mathrm{CO} 1-0$ for the blue and red components are presented in Figure 17(a). The spectra at the peak positions of the blue- and red-outflow components are also shown. The outflow map of $\mathrm{CO} 1-0$ made by integrating the intensity over the determined outflow wings is presented in Figure 17(b). The outflow wing emission is elongated mainly in the east-west $(\mathrm{E}-\mathrm{W})$ direction, and it also shows asymmetry in intensity and morphology. For example, the red component of the $\mathrm{CO} 1-0$ outflow is more broadly extended than the blue component. Also, the blue peak is located to the east of L1251A, and the red peak is located to the north of that region.

In comparison, an $\mathrm{HCO}^{+} 1-0$ outflow map shows the blue and red components with significant asymmetry in their intensity and size. The velocity ranges for the blue- and redshifted outflow wings of $\mathrm{HCO}^{+} \quad 1-0$ are -9.7 to $-5.9 \mathrm{~km} \mathrm{~s}^{-1}$ and -4.0 to $0.2 \mathrm{~km} \mathrm{~s}^{-1}$, respectively. Figure 18 shows that the blue component is dominant. A poorly collimated outflow of $\mathrm{HCO}^{+} 1-0$ is extended along the NW$\mathrm{SE}$ direction, i.e., inconsistent with the $\mathrm{E}-\mathrm{W}$ direction of the $\mathrm{CO} 1-0$ outflow. The position angle of the $\mathrm{HCO}^{+} 1-0$ outflow axis, however, does correspond to that of the $\mathrm{HCO}^{+}$ 1-0 outflow-like structure seen by Nikolić et al. (2003).

Figure 19 shows the outflow of CO 2-1 detected with the SMA. The velocity ranges for the blue- and redshifted outflow wings of CO 2-1 are -20 to $-7 \mathrm{~km} \mathrm{~s}^{-1}$ and from -2 to $10 \mathrm{~km} \mathrm{~s}^{-1}$, respectively. Then, the axis of this small-scale outflow is not consistent with either the CO $1-0$ outflow or the $\mathrm{HCO}^{+} 1-0$ outflow. The blue- and redshifted emission extends along the NE-SW direction and is very compact. IRS 1 is located at the position of the red peak of the $\mathrm{CO}$ 2-1 outflow and is closest to the center of the blue and red components. In addition, IRS 1 is in the earliest evolutionary stage of the three YSOs of L1251A detected in IRAC observations (see 5.1 below), suggesting that IRS 1 may be the driving source of the compact CO 2-1 outflow. However, in spite of no corresponding IR source, a radio jet has been detected in VLA 10 and its propagation direction is consistent with the compact outflow direction. Therefore, higher-resolution observations are needed to determine the engine of this compact outflow.

Different molecular outflows on different scales show dissimilarities in axis, morphology, and strength (Figures 1719), suggesting that there are multiple outflows from L1251A. The extended CO 1-0 outflow from Sato \& Fukui (1989, see their Figure 3) has a direction completely opposite that of the compact $\mathrm{CO} 2-1$ outflow presented here. The $\mathrm{HCO}^{+} 1-0$ and CO 1-0 outflows presented here may show a mixture of extended and compact outflows with poor collimation.

\section{DISCUSSION}

\subsection{Star Formation in Small Groups}

In L1251-C, 19 YSOs are detected, including 3 that are identified in the HDR mode observations. The submillimeter source, VLA 10, is excluded from this analysis because VLA 10 was not detected by Spitzer observations. $16 \%$ of sources are identified as Class 0/I YSOs and $84 \%$ of sources are identified as Class II, based on $T_{\text {bol }}$ classification (see Table 6). In L1251A, however, one is a Class 0/I source and two others are Class II sources. The whole field size of the MIPS observations of L1251-C is $\sim 1.44 \mathrm{pc}^{2}$, and therefore the surface density of YSOs in L1251-C is $\sim 13.2 \mathrm{pc}^{-2}$. The surface density of YSOs in L1251A is larger than that of the whole L1251-C region by two orders of magnitude. The area used to calculate the YSO surface density of L1251A is $\sim 0.08 \mathrm{pc}^{2}$, which covers its emission at submillimeter wavelengths (see Figure 3 ).

We compare L1251-C with the other core in L1251 (Sato et al. 1994) and also observed with Spitzer (Lee et al. 2006). Lee et al. (2006) shows that the surface density of YSOs in L1251-E is close to those of active star-forming regions. L1251-E $\left(\sim 2.5 \mathrm{pc}^{2}\right)$ has a larger area than L1251-C $\left(\sim 1.44 \mathrm{pc}^{2}\right)$. In L1251-E, 20 YSO candidates (4 Class 0/I, 14 Class II, and 3 unclassified YSOs) were detected in the MIPS $24 \mu \mathrm{m}$ band. As a result, in L1251-E, the surface density of YSOs is $\sim 10 \mathrm{pc}^{-2}$, which is slightly lower than that of L1251-C by a factor of 1.3. L1251B, the densest part of L1251-E, however, shows a larger YSO surface density than that of L1251A by a factor of two. The compositions of YSOs in various evolutionary stages in L1251-E and L1251-C show similar fractions. For example, the fractions of Class 0/I candidates in L1251-C and L1251-E are about 16 and 22\%, respectively. The fraction of Class 0/I sources in L1251B $(50 \%)$, is slightly larger than that in L1251A ( 34\%). Overall, L1251-C has a star formation environment similar to L1251-E.

One Class 0/I YSO and two Class II YSOs are located in L1251A in the IRAC HDR images (see, e.g., Figure 1). The average projected distance among the 19 YSOs in L1251-C is about $136^{\prime \prime}$, while the average projected distance of three YSOs in L1251A is about 5.'6, suggesting clustering. The intensity peak of the single-dish submillimeter continuum emission, which is coincident with all three YSOs of L1251A, is located between all three YSOs, suggesting that three YSOs at different evolutionary stages all reside in a singe dense core. Interferometer submillimeter molecular line emission from L1251A observed with SMA, which shows a more detailed 


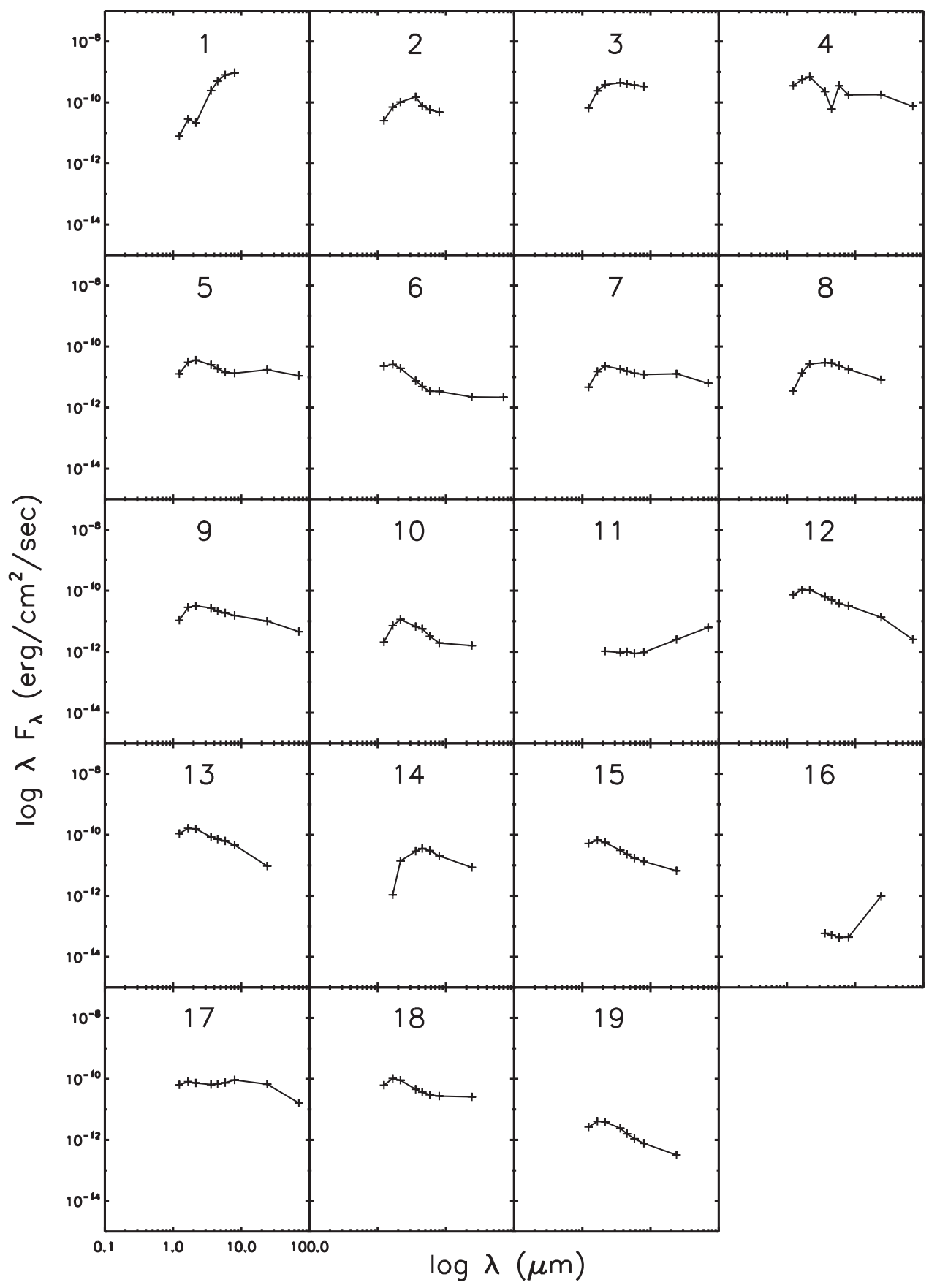

Figure 16. SED of the YSOs in L1251-C. The black lines connect observed fluxes.

view of the dense gas around the YSO group and resolves out the large r-scale structure, has intensity peaks located at the position of IRS 1, showing that IRS 1 is more deeply embedded than the other two YSOs. Single-dish submillimeter molecular line emission from L1251A, however, has integrated intensity peaks shifted to the south of $\mathrm{L} 1251 \mathrm{~A}$, probably because of CO evaporation.

The interferometric continuum data at $1.3 \mathrm{~mm}$ are likely tracing disks around protostars, but the contamination from the inner envelope cannot be ruled out because the deconvolved sizes of submillimeter sources are similar or slightly larger than the beam FWHM $(2 ! \cdot 7 \times 2 ! \cdot 5)$. The masses derived from the $1.3 \mathrm{~mm}$ continuum decrease in order of VLA 10, VLA 7, and
VLA $6\left(0.030,0.023\right.$, and $0.020 M_{\odot}$, respectively). VLA 10 does not have a corresponding IR source, indicating a deeply embedded source. Therefore, the $1.3 \mathrm{~mm}$ continuum emission of VLA 10 might trace its very dense inner envelope.

\subsection{Outflows in L1251A}

At least two outflows with different properties exist in L1251-C. One is an extended CO 1-0 outflow (Sato \& Fukui 1989) and the other is a compact CO $2-1$ outflow (this paper). The extended outflow from Sato \& Fukui (1989, see their Figure 3) shows a completely opposite outflow direction to that of the compact outflow from this work. The dynamical time of the extended outflow $\left(1.7 \times 10^{5} \mathrm{yr}\right)$ is greater than that 

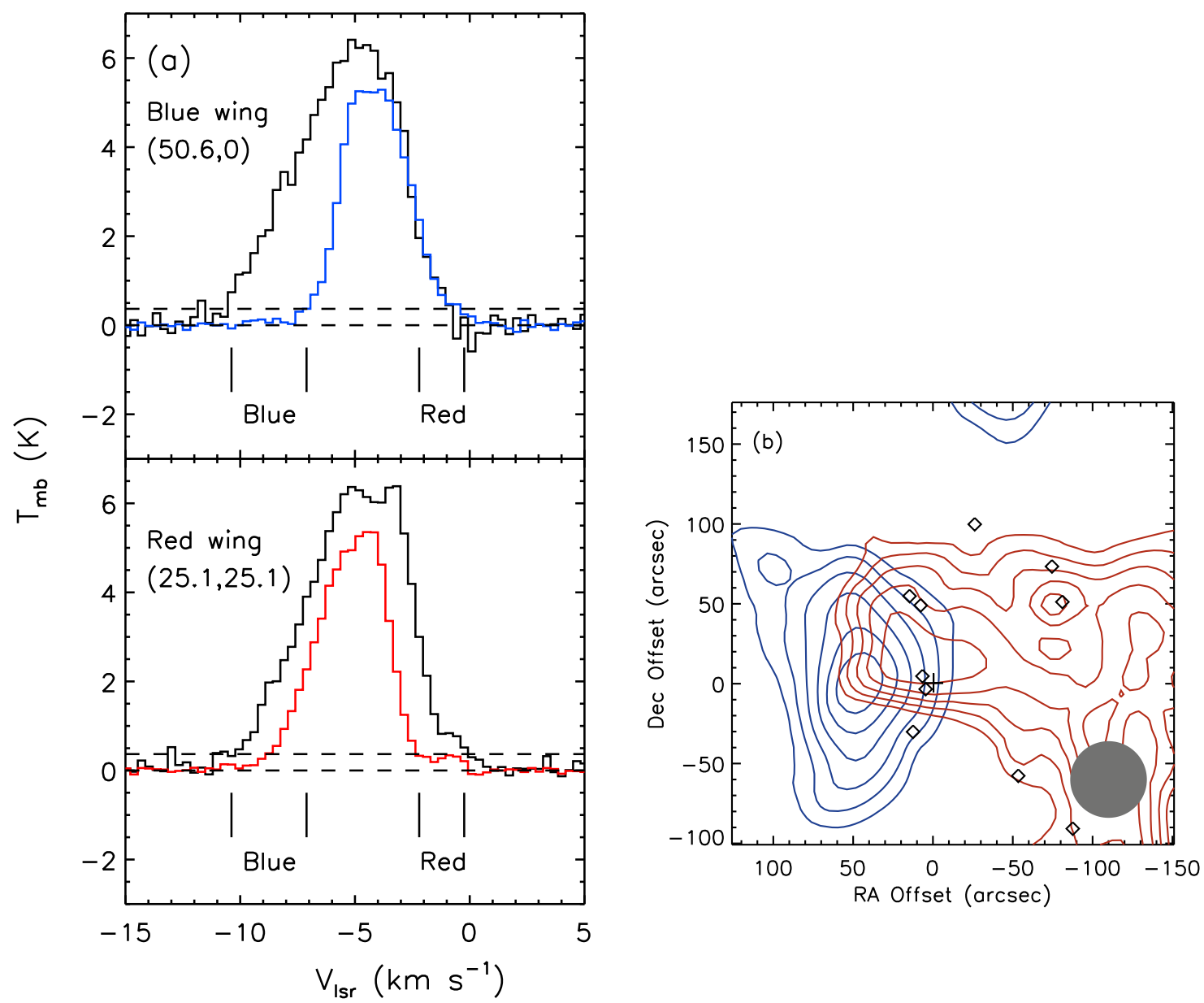

Figure 17. (a) The blue averaged spectrum of the blue wing free positions overlaid on the spectrum at the blueshifted emission peak position (top) and the red averaged spectrum of the red wing free positions overlaid on the spectrum at the redshifted emission peak position (bottom). (b) Integrated intensities of blue- and redshifted emission of $\mathrm{CO} 1-0$. Blue contours represent intensities integrated over the blueshifted range from systemic velocity. Red contours show intensities integrated over the redshifted range. The velocity range used for the blue and red components are from -10.4 to $-7.1 \mathrm{~km} \mathrm{~s}^{-1}$ and from -2.2 to $-0.2 \mathrm{~km} \mathrm{~s}^{-1}$, respectively. The contours range from $40 \%$ of the peak intensity to $90 \%$ and increase in steps of $10 \%$. IRS 1 and the other YSOs are denoted by a cross and diamonds, respectively. The beam FWHM is presented with a gray filled circle.

of the compact outflow $\left(4.7 \times 10^{3} \mathrm{yr}\right)$ by two orders of magnitude. The submillimeter continuum associated with radio jets (VLA 7 and VLA 10) is detected in L1251A. VLA 7 has a NW-SE propagation direction and VLA 10 has a NE-SW propagation direction. The poorly collimated distributions of the blue- and redshifted components of the $\mathrm{KVN} \mathrm{HCO}^{+}$ $1-0$ outflow and the TRAO CO 1-0 outflow may also be affected by the radio jets of VLA 7 and VLA 10.

The driving sources of each outflow, however, are hard to distinguish. A strong candidate of the driving source for the compact outflow is IRS 1 (VLA 6) because it is an embedded Class I source, and the blue- and redshifted lobes are closely associated with the position of IRS 1 (see Figure 19). However, VLA 10 cannot be ruled out because its dust-continuum emission seems to trace a very dense inner envelope (indicative of an extremely embedded source), and it is associated with a radio jet whose propagation direction is consistent with that of the compact outflow (Reipurth et al. 2004).

\section{SUMMARY}

L1251-C was observed as part of the Spitzer legacy project, c2d. Also, various molecular emission lines associated with L1251A have been observed with the KVN $21 \mathrm{~m}$ radio telescope and the TRAO $14 \mathrm{~m}$ radio telescope to understand its environment. Single-dish continuum data from other observations are also presented. In addition, SMA interferometric continuum and line data for L1251A were obtained at high angular resolution. With these data, we find the following.

1. Nineteen YSO candidates are associated with L1251-C, including three additionally identified in the HDR mode Spitzer IRAC bands: IRS 1, IRS 2, and IRS 3.

2. Single-dish continuum emission at 350 and $850 \mu \mathrm{m}$ is extended to the E-W and may trace an envelope containing all three sources. Individual condensations associated with each IR source are not resolved in the dust-continuum map at 350 and $850 \mu \mathrm{m}$. L1251A was mapped at $\mathrm{KVN}$ in $\mathrm{HCO}^{+} 1-0, \mathrm{H}^{13} \mathrm{CO}^{+} 1-0, \mathrm{~N}_{2} \mathrm{H}^{+} 1-0$, and $\mathrm{HCN} 1-0$. The integrated intensity peaks of all molecular lines observed at KVN are offset from the peak positions of the continuum maps at 350 and $850 \mu \mathrm{m}$. Notably, the integrated intensity maxima of $\mathrm{H}^{13} \mathrm{CO}^{+}$ $1-0$ and $\mathrm{N}_{2} \mathrm{H}^{+} 1-0$, which are optically thin, are offset by tens of arcseconds from L1251A to the south or southwest. The line profile of a well-known outflow and infall tracer $\mathrm{HCO}^{+} 1-0$ line are composed of two velocity (broad and narrow) components and show a self- 


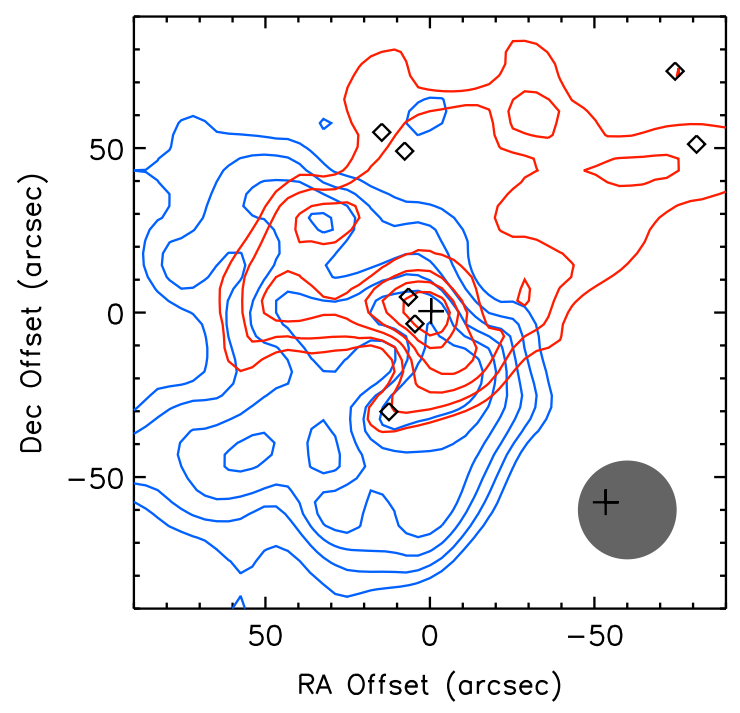

Figure 18. Integrated intensities of blue- and redshifted emission of $\mathrm{HCO}^{+} 1-0$. Blue contours represent intensities integrated over the blueshifted range from systemic velocity. Red contours show intensities integrated over the redshifted range. The velocity ranges used for the blue and red components are from -9.7 to $-5.9 \mathrm{~km} \mathrm{~s}^{-1}$ and from -4.0 to $-0.2 \mathrm{~km} \mathrm{~s}^{-1}$, respectively. The contours range from $40 \%$ of the peak intensity to $90 \%$ and increase in steps of 10\%. IRS 1 and the other YSOs are denoted by a cross and diamonds, respectively. The beam FWHM is represented by the gray filled circle.

absorption feature. Though this feature may trace infall, the broad component is a sign of outflow and the narrow component may trace dense envelope material (like other molecular lines described here). Several velocity components of the $\mathrm{H}_{2} \mathrm{O} 22 \mathrm{GHz}$ maser have been detected during multi-epoch observations.

3. Three dust-continuum condensations (VLA 6, VLA 7, and VLA 10) are detected by the SMA observations at $1.3 \mathrm{~mm}$. Two of three three (VLA 6 and VLA 7) are detected at the positions of IR sources (IRS 1 and IRS 3, respectively) in L1251A. The positions of IRS 2 and VLA 10 are separated by $\sim 2^{\prime \prime}$, suggesting that they are different sources. Finally, the total number of protostars in L1251A comes to four by adding the most embedded source of VLA 10, which does not have a corresponding IR source. The SMA integrated intensity maps of CO 2-1 and ${ }^{13} \mathrm{CO}$ 2-1 show complicated distributions, although a compact outflow is clearly detected in $\mathrm{CO}$ $2-1$ in the NE-SW direction.

4. In L1251-C, IRS 1, IRS 11, and IRS 16 are identified as Class I, and the other 16 YSOs are identified as Class II. The surface density of L1251-C $\left(\sim 1.44 \mathrm{pc}^{2}\right)$ is similar to that of L1251-E $\left(\sim 2.5 \mathrm{pc}^{2}\right)$. The fraction of Class 0/I sources $(16 \%)$ in L1251-C is also similar to that seen in L1251-E (22\%).

5. Molecular outflows on different scales have been mapped in $\mathrm{HCO}^{+} 1-0$, $\mathrm{CO} 1-0$, and $\mathrm{CO} 2-1$. These outflows lack coincidence in axis, morphology, and strength, suggesting that there are two outflows from two sources. One is an extended CO 1-0 outflow from Sato \& Fukui (1989) and the other is the compact SMA CO 2-1 outflow described here. $\mathrm{KVN} \mathrm{HCO}^{+} 1-0$ and TRAO $\mathrm{CO}$ 1-0 observations may show a mixture of extended and compact outflows. IRS 1 (VLA 6) is likely the driving source of the compact outflow detected in higherresolution SMA CO 2-1 observations, but VLA 10

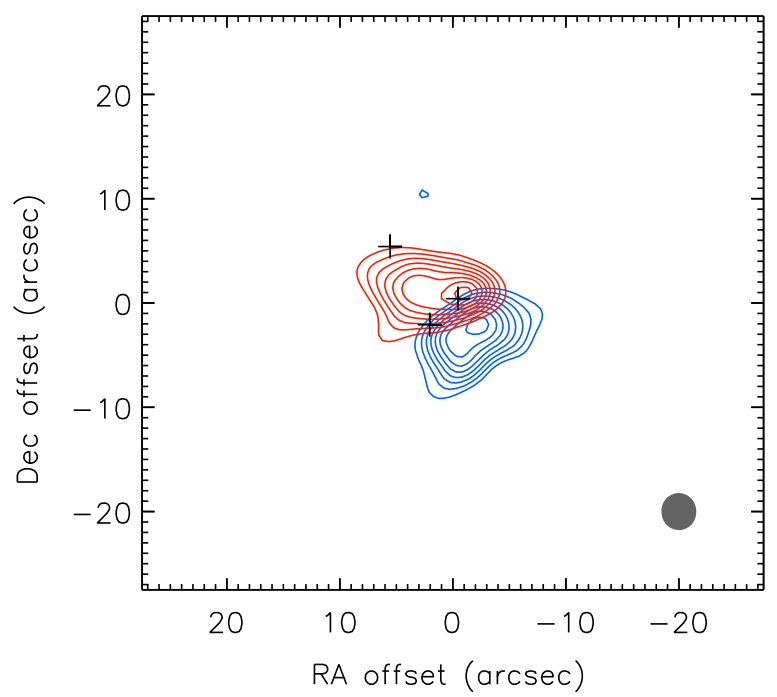

Figure 19. Integrated intensities of blue- and redshifted emission of CO 2-1. Blue contours represent the intensities integrated over the blueshifted range from -20 to $-7 \mathrm{~km} \mathrm{~s}^{-1}$ and red contours show the intensities integrated over the redshifted range from -2 to $10 \mathrm{~km} \mathrm{~s}^{-1}$. The contours range from $4 \sigma$ of the peak intensity to $90 \%$ and increase in steps of $10 \%$. VLA 6, VLA 7, and VLA 10 are denoted by crosses. The beam FWHM is represented by the gray filled ellipse.

cannot be ruled out. Therefore, interferometric observations with even higher spatial resolution are necessary to identify better which source is driving the compact outflow.

Support for this work, part of the Spitzer Legacy Science Program, was provided by NASA through contracts 1224608 and 1230782 issued by the Jet Propulsion Laboratory, California Institute of Technology, under NASA contract 1407. This research was also supported by the Basic Science Research Program through the National Research Foundation of Korea (NRF) funded by the Ministry of Education of the Korean government (grant No. NRF-2012R1A1A2044689). J.E.L. is very grateful to the department of Astronomy, University of Texas at Austin for the hospitality provided to her from 2013 August to 2014 July. This work was supported by the BK21 plus program through the National Research Foundation (NRF) funded by the Ministry of Education of Korea. This work was also supported by the Korea Astronomy and Space Science Institute (KASI) grant funded by the Korea government (MEST). This research was supported in part by the National Science Foundation under grant No. 0708158, and by NASA under grant No. NNX13AE54G (T.L.B.).

\section{REFERENCES}

Allen, L.-E., Calvet, N., D’Alessio, P., et al. 2004, ApJS, 154, 363 Anglada, G., Sepulveda, I., \& Gomez, J. F. 1997, A\&AS, 121, 255 Bae, J.-H., Kim, K.-T., Youn, S.-Y., et al. 2011, ApJS, 196, 21 Balazs, L. G., Eisloeffel, J., Holl, A., et al. 1992, A\&A, 255, 281 Beckwith, S. V. W., \& Sargent, A. I. 1991, ApJ, 381, 250 Beltrán, M. T., Estalella, R., Anglada, G., et al. 2001, AJ, 121, 1556 Bourke, T. L., Crapsi, A., Myers, P. C., et al. 2005, ApJL, 633, L129 Claussen, J. M., Wilking, A. B., Benson, J. P., et al. 1996, ApJS, 106, 111 Di Francesco, J., Johnstone, D., Kirk, H., et al. 2008, ApJS, 175, 277 Dunham, M. M., Evans, N. J., II, Bourke, T. L., et al. 2006, ApJ, 651, 945 Evans, N. J., II, Allen, L. E., Blake, G. A., et al. 2003, PASP, 115, 965 Evans, N. J., II, Harvey, P. M., Dunham, M. M., et al. 2007, Final Delivery of Data from the c2d Legacy Project: IRAC and MIPS, (Pasadena, CA: SSC) http://irsa.ipac.caltech.edu/data/SPITZER/docs/spitzermission/ observingprograms/legacy/ 
Evans, N. J., II, Dunham, M. M., Jørgensen, J. K., et al. 2009, ApJS, 181,321

Fazio, G. G., Hora, J. L., Allen, L. E., et al. 2004, ApJS, 154, 10

Han, S. T., Lee, J.-W., Kang, J., et al. 2008, IJIMW, 29, 69

Hatchell, J., Wilson, T., Drabek, E., et al. 2013, MNRAS, 429, L10

Hildebrand, R. H. 1983, QJRAS, 24, 267

Ho, P. T. P., Moran, J. M., \& Lo, K. Y. 2004, ApJL, 616, L1

Hodapp, K.-W. 1994, ApJS, 94, 615

Jørgensen, J. K., Bourke, T. L., Myers, P. C., et al. 2007, ApJ, 659, 479

Kun, M., Balog, Z., Kenyon, S. J., et al. 2009, ApJS, 185, 451

Kun, M., \& Prusti, T. 1993, A\&A, 272, 235

Lada, C. J., \& Lada, E. A. 2003, ARA\&A, 41, 57

Lee, J.-E., Di Francesco, J., Bourke, T. L., et al. 2007, ApJ, 671, 1748

Lee, J.-E., Di Francesco, J., Lai, S.-P., et al. 2006, ApJ, 648, 491

Lee, J.-E., Lee, H.-G., Shinn, J.-H., et al. 2010, ApJL, 709, L74

Lonsdale, C. J., Smith, H. E., Rowan-Robinson, M., et al. 2003, PASP, 115,897

Looney, L. W., Mundy, L. G., \& Welch, W. J. 2000, ApJ, 529, 477

Meehan, L. S. G., Wilking, B. A., Claussen, M. J., et al. 1998, AJ, 115, 1599
Myers, P. C., \& Ladd, E. F. 1993, ApJL, 413, L47

Nikolić, S., Johansson, L. E. B., \& Harju, J. 2003, A\&A, 409, 941

Reipurth, B., Rodríguez, L. F., Anglada, G., et al. 2004, AJ, 127, 1736

Rieke, G. H., Young, E. T., Engelbracht, C. W., et al. 2004, ApJS, 154, 25

Rosvick, J. M., \& Davidge, T. J. 1995, PASP, 107, 49

Sato, F., \& Fukui, Y. 1989, ApJ, 343, 773

Sato, F., Mizuno, A., Nagahama, T., et al. 1994, ApJ, 435, 279

Sault, R. J., Teuben, P. J., \& Wright, M. C. H. 1995, in ASP Conf. Ser. 77,

Astronomical Data Analysis Software and Systems IV, ed. R. A. Shaw,

H. E. Payne, \& J. J. E. Hayes (San Francisco, CA: ASP), 443

Schwartz, P. R., Gee, G., \& Huang, Y.-L. 1988, ApJ, 327, 350

Toth, V. L., \& Walmsley, C. M. 1994, IBVS, 4107, 1

Toth, V. L., \& Walmsley, C. M. 1996, A\&A, 311, 981

Wilking, B. A., Claussen, M. J., Benson, P. J., et al. 1994, ApJL, 431, L119

Wu, J., Dunham, M. M., Evans, N. J., II, et al. 2007, AJ, 133, 1560

Xiang, D., \& Turner, B. E. 1995, ApJS, 99, 121

Young, C. H., Bourke, T. L., Young, K. E., et al. 2006, AJ, 132, 1998

Young, C. H., Jørgensen, J. K., Shirley, Y. L., et al. 2004, ApJS, 154, 396

Zhou, S., Evans, N. J., II, Koempe, C., et al. 1993, ApJ, 404, 232 